\title{
CONTRÔLE PAR LES COEFFICIENTS DANS LE MODÈLE ELROD-ADAMS
}

\author{
Mohamed El Alaoui Talibi ${ }^{1}$ et Abdellah El Kacimi ${ }^{1}$
}

\begin{abstract}
The purpose of this paper is to study a control by coefficients problem issued from the elastohydrodynamic lubrication. The control variable is the film thickness. The cavitation phenomenon takes place and described by the Elrod-Adams model, suggested in preference to the classical variational inequality due to its ability to describe input and output flow. The idea is to use the penalization in the state equation by approximating the Heaviside graph whith a sequence of monotone and regular functions. We derive a necessary condition for the regularized problem, then we establish estimates of the state and the adjoint state in the one dimensional case. Next we pass to the limit.
\end{abstract}

Résumé. Dans ce papier, nous étudions un problème de contrôle par les coefficients issu de la lubrification élastohydrodynamique. La variable de contrôle est l'épaisseur du fluide. Le phénomène de cavitation est pris en compte par le modèle Elrod-Adams, connu pour ses performances dans la conservation des débits d'entrée et de sortie. L'idée est de régulariser dans l'équation d'état le graphe d'Heaviside, en l'approchant par une suite de fonctions monotones et régulières. Nous dérivons les conditions d'optimalité pour le problème régularisé, puis nous établissons des estimations de l'état et de l'état adjoint dans le cas unidimensionnel, ensuite nous passons à la limite.

Classification mathématique. 49J40, 76M30, 35R35, 76D08.

Reçu le 26 octobre 1999. Révisé le 26 mai et le 30 octobre 2000.

\section{INTRODUCTION}

Dans ce travail, nous étudions un problème de contrôle optimal dans le cadre des problèmes inverses en lubrification hydrodynamique. Connaissant la pression qui assure une bonne lubrification du contact, l'objectif est de chercher la forme du mécanisme, c'est-à-dire l'épaisseur du fluide correspondante.

Dans [17], on trouve une première motivation utilisant les techniques de contrôle optimal pour le palier unidimensionnel. Cependant, le phénomème de cavitation n'est pas pris en considération. Une approche dans ce sens apparaît dans [6], la cavitation étant prise en compte par le modèle de Reynolds [4], en utilisant une technique de pénalisation adaptée suivant les idées introduites dans [2,3].

Dans l'étude que nous présentons ici, on prend en compte la cavitation par le modèle Elrod-Adams [14], qui consiste à introduire dans les équations de Reynolds une variable supplémentaire modélisant le pourcentage de l'huile dans le film. Elle est liée à la pression d'une manière non linéaire et non différentiable. Ainsi, le problème de contrôle devient très difficile à résoudre. En effet, le critère, considéré comme une fonction de l'épaisseur, n'est même pas localement lipschitzien. L'idée fondamentale pour résoudre ce problème est d'approcher, par analogie avec $[2,6]$, le graphe d'Heaviside par une suite de fonctions monotones et continûment différentiables.

Mots-clés et phrases: Contrôle optimal, identification des coefficients, lubrification, problème elliptique non linéaire, régularisation.

1 Faculté des Sciences Semalila, Département de Mathématiques, BP. 2930 Marrakech, Maroc ; e-mail : elalaoui@ucam.ac.ma 
Nous décrivons brièvement le plan de ce travail : dans la section suivante, nous donnons une formulation du problème de contrôle. La troisième est consacrée à l'étude de l'existence d'un contrôle optimal. Dans la quatrième, nous formulons le problème de contrôle régularisé, puis nous dérivons les conditions nécessaires d'optimalité. La cinquième traite le comportement asymptotique de l'équation d'état régularisée. Dans la dernière section, nous établissons des estimations a priori permettant de passer à la limite, ensuite nous dérivons les conditions nécessaires d'optimalité (CNO) pour le problème initial dans le cas unidimensionnel. Pour cela, nous faisons une hypothèse sur la pression d'alimentation, que l'on considère strictement positive, dans le but de garantir une condition "d'intérieur non vide", puis nous adaptons une technique utilisée généralement pour dériver les CNO de type Fritz-John (non qualifié) pour des problèmes de contrôle avec contraintes sur l'état [11-13].

\section{Formulation du PRoblème de Contrôle}

Soit $\Omega$ un ouvert borné de $\mathbb{R}^{N}$, avec $N \leq 2$, de frontière régulière $\Gamma$ constituée de deux morceaux fermés et disjoints $\Gamma_{i}$ et $\Gamma_{e}$.

L'état du système est solution du problème non linéaire :

$$
\left(\mathcal{P}_{h}\right)\left\{\begin{array}{l}
\text { trouver }(\theta, p) \in L^{\infty}(\Omega) \times H^{1}(\Omega) \text { tel que } \\
-\operatorname{div}\left(h^{3} \nabla p\right)+\frac{\partial \theta h}{\partial x_{1}}=0 \quad \text { dans } \\
\theta \in H(p) \\
p \mid \Gamma_{i}=p_{\text {in }} \text { et } p \mid \Gamma_{e}=p_{\text {ex }}
\end{array}\right.
$$

où $p$ est la pression dans le film, $p_{\text {in }}$ et $p_{\text {ex }}$ désignent des pressions d'alimentation positives telles que $\left(p_{\text {in }}, p_{\text {ex }}\right)$ $\in \mathbb{R}_{+}^{*} \times \mathbb{R}_{+}, h$ l'épaisseur du film, $\theta$ la saturation et $H$ le graphe d'Heaviside défini par

$$
H(z)=\left\{\begin{array}{lll}
1 & \text { si } & z>0 \\
{[0,1]} & \text { si } & z=0 \\
0 & \text { si } & z<0
\end{array}\right.
$$

notons d'abord que l'existence et l'unicité pour ce problème sont obtenues dans [1,5] dans le cas du palier bidimensionnel.

Étant donnée une pression désirée $p_{d}$ appartenant à $L^{2}(\Omega)$, notre objectif est d'étudier le problème de contrôle optimal suivant :

$$
(\mathcal{M})\left\{\begin{array}{l}
\text { minimiser } J(h)=\frac{1}{2} \int_{\Omega}\left(p(h)-p_{d}\right)^{2} d x \\
h \in U_{\mathrm{ad}},
\end{array}\right.
$$

$p(h)$ étant la solution de $\left(\mathcal{P}_{h}\right)$ et $U_{\text {ad }}$ l' ensemble des contrôles admissibles :

$$
U_{\mathrm{ad}}:=\left\{h \in W^{1, r}(\Omega) \cap L^{\infty}(\Omega) / 0<a \leq h \leq b \text { et }\|\nabla h\|_{\left(L^{r}(\Omega)\right)^{N}} \leq c\right\},
$$

où $c$ est une constante et $r$ un réel tels que $c>0$ et $1<r \leq+\infty$. L'espace de Sobolev $W^{1, r}(\Omega)$ est défini par

$$
W^{1, r}(\Omega):=\left\{v \in L^{r}(\Omega) / \nabla v \in\left(L^{r}(\Omega)\right)^{N}\right\} .
$$


Remarque 2.1. Pour tout $r>1, U_{\mathrm{ad}}$ est un convexe fermé borné de $W^{1, r}(\Omega)$.

Remarque 2.2. Soit $\left\{h_{n}\right\}$ une suite bornée dans $L^{\infty}(\Omega)$. Alors $\left\{h_{n}\right\}$ converge dans $L^{1}(\Omega)$ si et seulement elle converge dans $L^{\lambda}(\Omega)$, pour $\lambda$ quelconque dans $[1,+\infty[$.

\section{RÉsultat D'existence}

Dans ce paragraphe, nous prouvons que le problème $(\mathcal{M})$ admet au moins une solution. Dans la suite, $\kappa_{A}$ désigne la fonction indicatrice de l'ensemble $A$. Nous commençons d'abord par montrer le résultat auxiliaire :

Proposition 3.1. Soit h appartenant à $U_{\mathrm{ad}}$ et $(\theta, p)$ la solution de $\left(\mathcal{P}_{h}\right)$; alors

$$
\begin{gathered}
\|p\|_{H^{1}(\Omega)} \leq C, \\
p \geq 0, \quad \text { p.p. dans } \Omega,
\end{gathered}
$$

où $C$ est une constante qui ne dépend pas de $h$.

Preuve. Soit $\psi$ appartenant à $H^{1}(\Omega)$ un relèvement des conditions aux limites. Prenons $p-\psi$ comme fonction test dans $\left(\mathcal{P}_{h}\right)$. En utilisant l'inégalité de Cauchy-Schwartz, nous obtenons :

$$
\begin{aligned}
a^{3} \int_{\Omega}|\nabla(p-\psi)|^{2} d x & \leq \int_{\Omega} h^{3}|\nabla(p-\psi)|^{2} d x \leq \int_{\Omega} \theta h \frac{\partial(p-\psi)}{\partial x_{1}} d x-\int_{\Omega} h^{3} \nabla \psi \cdot \nabla(p-\psi) d x \\
& \leq b\left\|\frac{\partial(p-\psi)}{\partial x_{1}}\right\|_{L^{1}(\Omega)}+b^{3}\|\nabla \psi\|_{\left(L^{2}(\Omega)\right)^{N}}\|\nabla(p-\psi)\|_{\left(L^{2}(\Omega)\right)^{N}} \\
& \leq\|\nabla(p-\psi)\|_{\left(L^{2}(\Omega)\right)^{N}}\left(b \operatorname{mes}(\Omega)^{\frac{1}{2}}+b^{3}\|\nabla \psi\|_{\left(L^{2}(\Omega)\right)^{N}}\right) ;
\end{aligned}
$$

par conséquent

$$
\|\nabla(p-\psi)\|_{\left(L^{2}(\Omega)\right)^{N}} \leq \frac{1}{a^{3}}\left(b \operatorname{mes}(\Omega)^{\frac{1}{2}}+b^{3}\|\nabla \psi\|_{\left(L^{2}(\Omega)\right)^{N}}\right)
$$

comme $p-\psi$ appartient à $H_{0}^{1}(\Omega)$, nous avons

$$
\begin{aligned}
\|p\|_{H^{1}(\Omega)} & \leq\|p-\psi\|_{H^{1}(\Omega)}+\|\psi\|_{H^{1}(\Omega)} \leq c\|\nabla(p-\psi)\|_{\left(L^{2}(\Omega)\right)^{N}}+\|\psi\|_{H^{1}(\Omega)} \\
& \leq \frac{c}{a^{3}}\left(b \operatorname{mes}(\Omega)^{\frac{1}{2}}+b^{3}\|\nabla \psi\|_{\left(L^{2}(\Omega)\right)^{N}}\right)+\|\psi\|_{H^{1}(\Omega)} .
\end{aligned}
$$

Pour montrer (2), on choisit $p^{-}=\max (0,-p)$, qui appartient à $H_{0}^{1}(\Omega)$, du fait que $p$ est positive sur $\Gamma$, comme fonction test dans $\left(\mathcal{P}_{h}\right)$. Comme $\kappa_{(p<0)} \theta=0$, presque partout dans $\Omega$, on obtient :

$$
-\int_{\Omega} h^{3}\left|\nabla p^{-}\right|^{2} d x=\int_{\Omega} \theta h \frac{\partial p^{-}}{\partial x_{1}} d x=-\int_{\Omega} \kappa_{(p<0)} \theta h \frac{\partial p}{\partial x_{1}} d x=0 .
$$

Ce qui achève la démonstration de la proposition.

Dans la suite, on notera

$$
\begin{aligned}
V_{i e} & =\left\{v \in H^{1}(\Omega) / v \mid \Gamma_{i}=p_{\text {in }} \text { et } v \mid \Gamma_{e}=p_{\mathrm{ex}}\right\}, \\
\Omega_{+} & =\{x \in \Omega / p(x)>0, \text { p.p. dans } \Omega\}
\end{aligned}
$$


et

$$
\Omega_{0}=\{x \in \Omega / p(x)=0, \text { p.p. dans } \Omega\}
$$

Grâce à l'estimation (1), on montre :

Proposition 3.2. Soit $\left(h_{n}\right)_{n}$ une suite d'éléments de $U_{\mathrm{ad}}$ et $\left(\theta_{n}, p_{n}\right)$ la solution de $\left(\mathcal{P}_{h_{n}}\right)$. Si

$$
\begin{gathered}
h_{n} \longrightarrow h \text { dans } L^{r}(\Omega), \\
h_{n} \longrightarrow h \text { dans } W^{1, r}(\Omega)-\text { faible }
\end{gathered}
$$

alors $h$ appartient à $U_{\mathrm{ad}}$; de plus, il existe une sous-suite de $\left(\theta_{n}, h_{n}, p_{n}\right)_{n}$ notée de la même façon et $(\theta, p)$ dans $L^{\infty}(\Omega) \times H^{1}(\Omega)$ tels que

$$
\begin{gathered}
h_{n} \longrightarrow h \text { dans } L^{\lambda}(\Omega), \forall \lambda \in[1,+\infty[, \\
\theta_{n} \longrightarrow \theta \text { dans } L^{\infty}(\Omega)-\text { faible }-*, \\
p_{n} \longrightarrow p \text { dans } H^{1}(\Omega)-\text { faible }
\end{gathered}
$$

où $(\theta, p)$ est la solution de $\left(\mathcal{P}_{h}\right)$.

Preuve. $U_{\text {ad }}$ est un convexe fermé de $W^{1, r}(\Omega)$, chaque élément de la suite $\left\{h_{n}\right\}$ appartenant à $U_{\text {ad }}$, (3) et $(4)$ entraînent que $h$ appartient à $U_{\text {ad }}$. La conclusion (5) découle de (3) et la remarque 2.2. Quant à (6), elle résulte du fait que $0 \leq \theta_{n} \leq 1$, presque partout dans $\Omega$. D'après (1), la suite $\left\{p_{n}\right\}$ étant bornée dans $H^{1}(\Omega)$, on a $(7)$. Il reste à montrer que $(\theta, p)$ est solution de $\left(\mathcal{P}_{h}\right)$. En utilisant la définition de $\left(\mathcal{P}_{h_{n}}\right)$ avec une fonction test $\varphi$ appartenant à $D(\Omega)$, et compte tenu de $(5,6)$ et $(7)$, on montre, par passage à la limite sur $n$, que

$$
\int_{\Omega} h^{3} \nabla p \nabla \varphi-\theta h \frac{\partial \varphi}{\partial x_{1}} d x=0, \quad \forall \varphi \in D(\Omega)
$$

$D(\Omega)$ étant dense dans $H_{0}^{1}(\Omega)$, l'égalité $(8)$ reste vraie pour toute fonction test $\varphi$ de $H_{0}^{1}(\Omega)$. L'inclusion de $H^{1}(\Omega)$ dans $L^{2}(\Omega)$ est compacte, donc (1) implique que

$$
p_{n} \longrightarrow p \text { dans } L^{2}(\Omega)
$$

on vérifie aisément, pour presque tout $x$ de $\Omega$, que

$$
\theta_{n} \in H\left(p_{n}\right) \text { et } p_{n} \geq 0 \Leftrightarrow\left(1-\theta_{n}\right) p_{n}=0, \theta_{n} \in[0,1] \text { et } p_{n} \geq 0 ;
$$

donc, en faisant tendre $n$ vers $+\infty$, (6) et (9) entrainent que

$$
(1-\theta) p=0, p \geq 0 \text { et } \theta \in[0,1], \text { p.p. dans } \Omega
$$

d'où $\theta \in H(p)$. L'application trace $\gamma_{0}$, définie de $H^{1}(\Omega)$ dans $L^{2}(\Gamma)$, étant continue, $V_{i e}$ est un convexe fermé de $H^{1}(\Omega)$. Et comme $\left\{p_{n}\right\}$ est une suite d'éléments de $V_{i e}$, il s'ensuit de $(7)$ que $p$ appartient à $V_{i e}$. 
Nous terminons cette section par le théorème d'existence suivant :

Théorème 3.3. Il existe au moins un contrôle optimal $h^{*}$ pour le problème $(\mathcal{M})$.

Preuve. Soit $\left\{h_{n}\right\}$ une suite minimisante. Pour tout $r$ dans $\left.] 1, \infty\right]$, l'inclusion de $W^{1, r}(\Omega)$ dans $L^{r}(\Omega)$ étant compacte, on peut extraire une sous-suite $\left\{h_{n_{k}}\right\}_{k}$ telle que $h_{n_{k}}$ converge vers $h^{*}$ dans $L^{r}(\Omega)$ et $W^{1, r}(\Omega)$-faible ; d'après la proposition 3.2 et $(9), h^{*}$ appartient à $U_{\text {ad }}$ et $\left\{p_{n_{k}}\right\}$ converge vers $p^{*}$ dans $L^{2}(\Omega)$. Par conséquent

$$
\inf _{h \in U_{\text {ad }}} J(h)=\lim J\left(h_{n_{k}}\right)=J\left(h^{*}\right) .
$$

Donc $h^{*}$ est un contrôle optimal pour le problème $(\mathcal{M})$.

\section{Conditions néCessaires d’optimalité pour le problème $(\mathcal{M})_{\varepsilon}$}

Dans cette section, nous donnons le système d'optimalité pour le problème $(\mathcal{M})_{\varepsilon}$. Utilisant la démarche de $[2,3]$, on définit une suite de problèmes régularisés $\left(\mathcal{P}_{h}\right)_{\varepsilon}$ associés à $\left(\mathcal{P}_{h}\right)$ de la manière suivante :

$$
\left(\mathcal{P}_{h}\right)_{\varepsilon}\left\{\begin{array}{l}
p_{\varepsilon} \in H^{1}(\Omega) \\
-\operatorname{div}\left(h^{3} \nabla p_{\varepsilon}\right)+\frac{\partial\left(H_{\varepsilon}\left(p_{\varepsilon}\right) h\right)}{\partial x_{1}}=0 \\
p_{\varepsilon} \mid \Gamma_{i}=p_{\text {in }} \text { et } p_{\varepsilon} \mid \Gamma_{e}=p_{\text {ex }},
\end{array}\right.
$$

où $H_{\varepsilon}$ est une régularisation $C^{1}$ du graphe d'Heaviside définie par

$$
H_{\varepsilon}(z)= \begin{cases}1 & \text { si } z \geq \varepsilon \\ 0 & \text { si } \quad z \leq 0 \\ \frac{2 z^{2}}{\varepsilon^{2}} & \text { si } \quad 0 \leq z \leq \frac{\varepsilon}{2} \\ 1-2\left(\frac{z}{\varepsilon}-1\right)^{2} & \text { si } \frac{\varepsilon}{2} \leq z \leq \varepsilon\end{cases}
$$

On approche le problème $(\mathcal{M})$, comme dans [2], par :

$$
(\mathcal{M})_{\varepsilon}\left\{\begin{array}{l}
\operatorname{minimiser} J_{\varepsilon}(h)=\frac{1}{2} \int_{\Omega}\left(p_{\varepsilon}-p_{d}\right)^{2} d x+\frac{1}{2} \int_{\Omega}\left(h-h^{*}\right)^{2} d x \\
h \in U_{\mathrm{ad}},
\end{array}\right.
$$

$h^{*}$ étant un contrôle optimal pour $(\mathcal{M})$.

Remarque 4.1. Le terme de pénalisation adaptée $\frac{1}{2} \int_{\Omega}\left(h-h^{*}\right)^{2} d x$ est ajouté au critère (lorsque l'unicité n'a pas lieu) pour assurer la convergence forte dans $L^{2}(\Omega)$ de la suite des solutions de $(\mathcal{M})_{\varepsilon}$ vers un contrôle optimal $h^{*}$ donné [2, 3].

Remarque 4.2. En dérivant $H_{\varepsilon}$ on obtient, pour $z$ appartenant à $\mathbb{R}$,

$$
\dot{H}_{\varepsilon}(z)=\frac{4 z}{\varepsilon^{2}} \kappa\left(\left\{0 \leq z \leq \frac{\varepsilon}{2}\right\}\right)-\frac{4}{\varepsilon}\left(\frac{z}{\varepsilon}-1\right) \kappa\left(\left\{\frac{\varepsilon}{2} \leq z \leq \varepsilon\right\}\right) .
$$


La fonction $H_{\varepsilon}$ vérifie les propriétés suivantes :

$$
\begin{gathered}
0 \leq\left(H_{\varepsilon}(z)-H_{\varepsilon}(\bar{z})\right)(z-\bar{z}) \leq \frac{2}{\varepsilon}(z-\bar{z})^{2}, \\
\left|\dot{H}_{\varepsilon}(z)-\dot{H}_{\varepsilon}(\bar{z})\right| \leq \frac{4}{\varepsilon^{2}}|z-\bar{z}|,
\end{gathered}
$$

pour $z$ et $\bar{z}$ quelconques dans $\mathbb{R}$.

Nous commençons par montrer que le problème $\left(\mathcal{P}_{h}\right)_{\varepsilon}$ admet une solution unique. Nous allons utiliser le théorème du point fixe de Schauder afin de prouver l'existence pour ce problème non linéaire.

Proposition 4.3. Pour tout $h$ dans $U_{\mathrm{ad}}$, le problème $\left(\mathcal{P}_{h}\right)_{\varepsilon}$ admet une solution unique $p_{\varepsilon}$ vérifiant

$$
p_{\varepsilon} \geq 0, \quad \text { p.p. } \quad \text { dans } \Omega \text {. }
$$

Preuve. Nous allons montrer d'abord l'existence. Pour cela, considérons l'application $T_{\varepsilon}$ qui à un élément $q$ de $V_{i e}$ associe l'unique solution $\bar{p}_{\varepsilon}$ du problème elliptique linéaire

$$
\left\{\begin{array}{l}
\bar{p}_{\varepsilon} \in V_{i e} \\
-\operatorname{div}\left(h^{3} \nabla \bar{p}_{\varepsilon}\right)+\frac{\partial\left(h H_{\varepsilon}(q)\right)}{\partial x_{1}}=0 ;
\end{array}\right.
$$

nous rappelons que $V_{i e}$ est un convexe fermé de l'espace de sobolev $H^{1}(\Omega)$. Soient $q_{1}, q_{2}$ deux éléments de $V_{i e}, \bar{p}_{\varepsilon}^{1}$ et $\bar{p}_{\varepsilon}^{2}$ les solutions de (13) correspondantes. Faisons la différence de $(13)_{q_{1}}$ avec $(13)_{q_{2}}$ et prenons $\bar{p}_{\varepsilon}^{1}-\bar{p}_{\varepsilon}^{2}$ comme fonction test dans l'équation obtenue ; on montre que

$$
\left\|\nabla\left(\bar{p}_{\varepsilon}^{1}-\bar{p}_{\varepsilon}^{2}\right)\right\|_{\left(L^{2}(\Omega)\right)^{N}} \leq \frac{b}{a^{3}}\left\|H_{\varepsilon}\left(q_{1}\right)-H_{\varepsilon}\left(q_{2}\right)\right\|_{L^{2}(\Omega)} ;
$$

comme la fonction $H_{\varepsilon}$ est lipschitzienne de rapport $\frac{2}{\varepsilon}$, l'inégalité de Poincaré implique que

$$
\left\|T_{\varepsilon} q_{1}-T_{\varepsilon} q_{2}\right\|_{H^{1}(\Omega)} \leq C(a, b, N, \varepsilon, \Omega)\left\|q_{1}-q_{2}\right\|_{L^{2}(\Omega)} .
$$

La compacité de l'injection de $H^{1}(\Omega)$ dans $L^{2}(\Omega)$ permet de déduire de (14) que l'application $T_{\varepsilon}$ est complètement continue. En appliquant le théorème du point fixe de Shauder, nous concluons l'existence d'une solution pour $\left(\mathcal{P}_{h}\right)_{\varepsilon}$.

Il reste à montrer l'unicité. Soient $\bar{p}_{\varepsilon}^{1}$ et $\bar{p}_{\varepsilon}^{2}$ deux solutions et posons

$$
\varphi_{\mu}=\frac{q^{+}}{q^{+}+\mu}, \text { avec } q=\bar{p}_{\varepsilon}^{1}-\bar{p}_{\varepsilon}^{2}, q^{+}=\max (0, q) \text { et } \mu>0 .
$$

On fait la différence des deux équations vérifiées par $\bar{p}_{\varepsilon}^{1}$ et $\bar{p}_{\varepsilon}^{2}$, puis on multiplie l'équation obtenue par la fonction test $\varphi_{\mu}$, qui est dans $H_{0}^{1}(\Omega)$. On obtient, après simplification par $\mu$,

$$
\int_{\Omega}\left|\frac{\nabla\left(q^{+}+\mu\right)}{q^{+}+\mu}\right|^{2} d x \leq \frac{2 b}{a^{3} \varepsilon} \int_{\Omega} \frac{q^{+}}{q^{+}+\mu}\left|\frac{\nabla\left(q^{+}+\mu\right)}{q^{+}+\mu}\right| d x \leq \frac{2 b}{a^{3} \varepsilon} \int_{\Omega}\left|\frac{\nabla\left(q^{+}+\mu\right)}{q^{+}+\mu}\right| d x ;
$$


soit

$$
w_{\mu}=\log \left(1+\frac{q^{+}}{\mu}\right)
$$

comme $w_{\mu}$ appartient à $H_{0}^{1}(\Omega)$ et $\nabla w_{\mu}=\frac{\nabla\left(q^{+}+\mu\right)}{q^{+}+\mu}$, il s'ensuit en utilisant les inégalités de Cauchy-Schwartz et de Poincaré que

$$
\int_{\Omega}\left(\log \left(1+\frac{q^{+}}{\mu}\right)\right)^{2} d x \leq C
$$

où $C$ est une constante qui ne dépend pas de $\mu$.

Enfin, si on fait tendre $\mu$ vers 0 , nous aurons nécessairement $q \leq 0$, p.p. dans $\Omega$. En effet, remarquons d'abord que la suite $\left\{w_{\mu}^{2}\right\}$ converge presque partout vers la fonction $E$ définie par

$$
E(x)=\left\{\begin{array}{lll}
0 & \text { si } & q(x) \leq 0 \\
+\infty & \text { si } & q(x)>0
\end{array}\right.
$$

$\left\{w_{\mu}^{2}\right\}$ étant croissante, on peut alors appliquer, compte tenu de l'estimation (15), le théorème de convergence monotone de Beppo Levi et déduire que $\left\{w_{\mu}^{2}\right\}$ converge vers $E$ dans $L^{1}(\Omega)$. En passant à la limite sur $\mu$ dans (15), nous obtenons

$$
\int_{\Omega} E d x \leq C
$$

comme $E=+\infty$ sur $\{q>0\}$, on a $q \leq 0$, p.p. dans $\Omega$. En remplaçant $q$ par $-q$, on obtient aussi $q \geq$ 0 , p.p. dans $\Omega$; ce qui prouve l'unicité.

Pour montrer (12), il suffit d'utiliser, comme dans la démonstration de la proposition 3.1, la fonction test $p_{\varepsilon}^{-}$, qui est dans $H_{0}^{1}(\Omega)$.

À l'aide du théorème des fonctions implicites, nous allons montrer que l'application $h \longrightarrow p_{\varepsilon}(h)$ est de classe $C^{1}$ de $U_{\text {ad }}$ dans $H^{1}(\Omega)$. On introduit l'espace $X=H_{0}^{1}(\Omega) \times L^{\infty}(\Omega)$ muni de la norme $\|\cdot\|_{X}$ définie par

$$
\|(v, g)\|_{X}=\|v\|_{H_{0}^{1}(\Omega)}+\|g\|_{L^{\infty}(\Omega)}, \text { pour }(v, g) \text { dans } X .
$$

On note par $\mathcal{L}\left(X, H^{-1}(\Omega)\right)$ l'espace des applications linéaires continues de $X$ dans $H^{-1}(\Omega)$. Montrons d'abord le résultat suivant :

Proposition 4.4. Soit $(h, f)$ appartenant à $U_{\mathrm{ad}} \times H^{-1}(\Omega)$; alors le problème linéaire

$$
\left\{\begin{array}{l}
v_{\varepsilon} \in H_{0}^{1}(\Omega) \\
-\operatorname{div}\left(h^{3} \nabla v_{\varepsilon}\right)+\frac{\partial\left(h \dot{H}_{\varepsilon}\left(p_{\varepsilon}\right) v_{\varepsilon}\right)}{\partial x_{1}}=f \quad \text { dans } \quad H^{-1}(\Omega)
\end{array}\right.
$$

admet une solution unique.

Preuve. La difficulté principale du problème réside dans la non coercivité de la forme bilinéaire

$$
a_{\varepsilon}(v, w)=\int_{\Omega}\left(h^{3} \nabla v \cdot \nabla w-h \dot{H}_{\varepsilon}\left(p_{\varepsilon}\right) v \frac{\partial w}{\partial x_{1}}\right) d x .
$$


Nous allons d'abord montrer l'unicité. L'équation (16) étant linéaire, il suffit de prouver, en supposant $f=0$, que $v_{\varepsilon} \leq 0$, p.p. dans $\Omega$. En multipliant (16) par la fonction test $\frac{v_{\varepsilon}{ }^{+}}{v_{\varepsilon}+\mu}$, comme dans la démonstration de la proposition 4.3, on montre de la même façon que $v_{\varepsilon}{ }^{+}=0$.

Maintenant, considérons $\sigma>0$ convenable tel que l'opérateur linéaire $L_{\sigma}$ défini de $H_{0}^{1}(\Omega)$ dans $H^{-1}(\Omega)$ par

$$
L_{\sigma} v=\sigma v-\operatorname{div}\left(h^{3} \nabla v\right)+\frac{\partial\left(h \dot{H}_{\varepsilon}\left(p_{\varepsilon}\right) v\right)}{\partial x_{1}}
$$

soit coercif, autrement dit

$$
\left\langle L_{\sigma} v, v\right\rangle \geq \alpha\|v\|_{H_{0}^{1}(\Omega)}^{2}, \operatorname{avec} \alpha>0
$$

on peut choisir, pour $\sigma \geq \frac{2 b}{\varepsilon}, \alpha=C\left(\sigma-\frac{2 b}{\varepsilon}\right)+a^{3}$, où $C$ est la constante de Poincaré. Remarquons que $v_{\varepsilon}$ est solution de (16) si et seulement si

$$
v_{\varepsilon}-\sigma L_{\sigma}^{-1} \circ I v_{\varepsilon}=L_{\sigma}^{-1} f
$$

où $I$ est l'opérateur défini de $H_{0}^{1}(\Omega)$ dans $H^{-1}(\Omega)$ par

$$
\langle I v, w\rangle=\int_{\Omega} v w d x
$$

Comme l'inclusion de $H_{0}^{1}(\Omega)$ dans $L^{2}(\Omega)$ est compacte, $I$ est complètement continu [15]. De plus, l'opérateur $\sigma L_{\sigma}^{-1}$ est continu, donc $\sigma L_{\sigma}^{-1} \circ I$ est complètement continu. Il suffit maintenant d'appliquer l'alternative de Fredholm [9] pour conclure.

Soit $G_{\varepsilon}$ l'application définie de $X$ dans $H^{-1}(\Omega)$ par

$$
G_{\varepsilon}(w, h)=-\operatorname{div}\left(h^{3} \nabla(w+\psi)\right)+\frac{\partial\left(h H_{\varepsilon}(w+\psi)\right)}{\partial x_{1}}, \operatorname{pour}(w, h) \in X,
$$

$\psi$ étant un relèvement $H^{1}(\Omega)$ des conditions aux limites. On montre :

Proposition 4.5. L'application $G_{\varepsilon}$ est de classe $C^{1}$ de $X$ dans $H^{-1}(\Omega)$.

Preuve. Soit $(v, g)$ appartenant à $X$, avec $(v, g) \neq 0$. On vérifie facilement que

$$
\begin{aligned}
\delta(v, g):= & \frac{G_{\varepsilon}(w+v, h+g)-G_{\varepsilon}(w, h)-\left(L_{\varepsilon}(w, h), v, g\right)}{\|(v, g)\|_{X}} \\
= & \frac{1}{\|(v, g)\|_{X}} \times\left(-\operatorname{div}\left(\left((h+g)^{3}-h^{3}-3 h^{2} g\right) \nabla(w+\psi)\right)\right. \\
& -\operatorname{div}\left(\left((h+g)^{3}-h^{3}\right) \nabla v\right)+\frac{\partial\left(g\left(H_{\varepsilon}(w+v+\psi)-H_{\varepsilon}(w+\psi)\right)\right)}{\partial x_{1}} \\
& \left.+\frac{\partial\left(h\left(H_{\varepsilon}(w+v+\psi)-H_{\varepsilon}(w+\psi)-\dot{H}_{\varepsilon}(w+\psi) v\right)\right)}{\partial x_{1}}\right),
\end{aligned}
$$

où $L_{\varepsilon}$ est l'application définie de $X$ dans $\mathcal{L}\left(X, H^{-1}(\Omega)\right)$ par

$$
\left(L_{\varepsilon}(w, h), v, g\right)=-\operatorname{div}\left(3 h^{2} g \nabla(w+\psi)\right)+\frac{\partial\left(g H_{\varepsilon}(w+\psi)\right)}{\partial x_{1}}-\operatorname{div}\left(h^{3} \nabla v\right)+\frac{\partial\left(h \dot{H}_{\varepsilon}(w+\psi) v\right)}{\partial x_{1}} .
$$


Donc

$$
\begin{aligned}
\|\delta(v, g)\|_{H^{-1}(\Omega)} \leq & \frac{1}{\|(v, g)\|_{X}} \times\left(\left\|(h+g)^{3}-h^{3}-3 h^{2} g\right\|_{L^{\infty}(\Omega)}\|w+\psi\|_{H^{1}(\Omega)}\right. \\
& +\left\|(h+g)^{3}-h^{3}\right\|_{L^{\infty}(\Omega)}\|v\|_{H_{0}^{1}(\Omega)} \\
& +\|g\|_{L^{\infty}(\Omega)}\left\|H_{\varepsilon}(w+v+\psi)-H_{\varepsilon}(w+\psi)\right\|_{L^{2}(\Omega)} \\
& \left.+\|h\|_{L^{\infty}(\Omega)}\left\|H_{\varepsilon}(w+v+\psi)-H_{\varepsilon}(w+\psi)-\dot{H}_{\varepsilon}(w+\psi) v\right\|_{L^{2}(\Omega)}\right) ;
\end{aligned}
$$

en remarquant que

$$
(h+g)^{3}-h^{3}-3 h^{2} g=g^{3}+3 h g^{2},
$$

on établit les majorations suivantes

$$
\begin{gathered}
\left\|(h+g)^{3}-h^{3}-3 h^{2} g\right\|_{L^{\infty}(\Omega)} \leq O\left(\|g\|_{L^{\infty}(\Omega)}\right)\|g\|_{L^{\infty}(\Omega)}, \\
\left\|(h+g)^{3}-h^{3}\right\|_{L^{\infty}(\Omega)} \leq O\left(\|g\|_{L^{\infty}(\Omega)}\right),
\end{gathered}
$$

où $O(|z|)$ est tel que $\frac{O(|z|)}{|z|}$ reste borné quand $|z|$ tend vers 0 . De plus, (10) et l'inégalité de Poincaré impliquent que

$$
\left\|H_{\varepsilon}(w+v+\psi)-H_{\varepsilon}(w+\psi)\right\|_{L^{2}(\Omega)} \leq \frac{2}{\varepsilon}\|v\|_{L^{2}(\Omega)} \leq O\left(\|v\|_{H_{0}^{1}(\Omega)}\right)
$$

compte tenu de (11), le théorème des accroissements finis, appliqué à la fonction $H_{\varepsilon}$, entraîne que

$$
\left\|H_{\varepsilon}(w+v+\psi)-H_{\varepsilon}(w+\psi)-\dot{H}_{\varepsilon}(w+\psi) v\right\|_{L^{2}(\Omega)} \leq \frac{4}{\varepsilon^{2}}\|v\|_{L^{2}(\Omega)}^{2} \leq O\left(\|v\|_{H_{0}^{1}(\Omega)}\right)^{2} .
$$

Il s'ensuit des majorations (17-20) que

$$
\begin{aligned}
\|\delta(v, g)\|_{H^{-1}(\Omega)} \leq & \frac{1}{\|(v, g)\|_{X}} \times\left(O\left(\|g\|_{L^{\infty}(\Omega)}\right)\|g\|_{L^{\infty}(\Omega)}+O\left(\|g\|_{L^{\infty}(\Omega)}\right)\|v\|_{H_{0}^{1}(\Omega)}\right. \\
& \left.+\|g\|_{L^{\infty}(\Omega)} O\left(\|v\|_{H_{0}^{1}(\Omega)}\right)+O\left(\|v\|_{H_{0}^{1}(\Omega)}\right)^{2}\right) \leq O\left(\|(v, g)\|_{X}\right)
\end{aligned}
$$

par conséquent, l'application $G_{\varepsilon}$ est différentiable et $\nabla G_{\varepsilon}(w, h)=L_{\varepsilon}(w, h)$.

Enfin, par des arguments similaires, on montre que $\nabla G_{\varepsilon}$ est continu de $X$ dans $\mathcal{L}\left(X, H^{-1}(\Omega)\right)$.

Nous pouvons maintenant démontrer le résultat suivant :

Proposition 4.6. L'application $h \longrightarrow p_{\varepsilon}(h)$ est de classe $C^{1}$ de $U_{\mathrm{ad}}$ dans $H^{1}(\Omega)$.

Preuve. En posant $w_{\varepsilon}=p_{\varepsilon}-\psi$, la démonstration de la proposition 4.6 se ramène à prouver que l'application $h \longrightarrow w_{\varepsilon}(h)$ est continûment différentiable. D'après la définition de $p_{\varepsilon}(h)$, nous avons $G_{\varepsilon}\left(w_{\varepsilon}(h), h\right)=0$, pour tout $h$ de $U_{\text {ad }}$. La proposition 4.4, implique que l'opérateur $\nabla_{w} G_{\varepsilon}\left(w_{\varepsilon}(h), h\right)$ défini par

$$
\left\langle\nabla_{w} G_{\varepsilon}\left(w_{\varepsilon}(h), h\right), v\right\rangle=-\operatorname{div}\left(h^{3} \nabla v\right)+\frac{\partial\left(h \dot{H}_{\varepsilon}\left(w_{\varepsilon}(h)+\psi\right) v\right)}{\partial x_{1}}
$$


est un isomorphisme de $H_{0}^{1}(\Omega)$ dans $H^{-1}(\Omega)$; de plus, l'application $G_{\varepsilon}$ est de classe $C^{1}$. Nous pouvons alors appliquer le théorème des fonctions implicites pour aboutir à la conclusion cherchée.

Pour finir cette section, nous énonçons le théorème suivant qui donne les conditions nécessaires d'optimalité $\operatorname{pour}(\mathcal{M})_{\varepsilon}$ :

Théorème 4.7. Il existe au moins un contrôle optimal $h_{\varepsilon}^{*}$ pour le problème $(\mathcal{M})_{\varepsilon}$ vérifiant le système d'optimalité suivant:

$$
\begin{aligned}
& \left\{\begin{array}{l}
p_{\varepsilon}^{*} \in H^{1}(\Omega) \\
-\operatorname{div}\left(h_{\varepsilon}^{*^{3}} \nabla p_{\varepsilon}^{*}\right)+\frac{\partial\left(H_{\varepsilon}\left(p_{\varepsilon}^{*}\right) h_{\varepsilon}^{*}\right)}{\partial x_{1}}=0 \\
p_{\varepsilon}^{*} \mid \Gamma_{i}=p_{\text {in }} \text { et } p_{\varepsilon}^{*} \mid \Gamma_{e}=p_{\mathrm{ex}},
\end{array}\right. \\
& \forall h \in U_{\mathrm{ad}} \\
& \int_{\Omega}\left(h-h_{\varepsilon}^{*}\right)\left(H_{\varepsilon}\left(p_{\varepsilon}^{*}\right) \frac{\partial q_{\varepsilon}}{\partial x_{1}}-3 h_{\varepsilon}^{* 2} \nabla p_{\varepsilon}^{*} \nabla q_{\varepsilon}+h_{\varepsilon}^{*}-h^{*}\right) d x \geq 0,
\end{aligned}
$$

où $q_{\varepsilon}$ est la solution de l'équation d'état adjointe :

$$
\left\{\begin{array}{l}
q_{\varepsilon} \in H_{0}^{1}(\Omega) \\
-\operatorname{div}\left(h_{\varepsilon}^{* 3} \nabla q_{\varepsilon}\right)-h_{\varepsilon}^{*} \dot{H}_{\varepsilon}\left(p_{\varepsilon}^{*}\right) \frac{\partial q_{\varepsilon}}{\partial x_{1}}=p_{\varepsilon}^{*}-p_{d}
\end{array}\right.
$$

Preuve. Pour prouver que $(\mathcal{M})_{\varepsilon}$ admet au moins une solution, on raisonne de la même manière que dans la section (3).

On commence d'abord par montrer l'unicité de la solution de (23) ; puis on raisonne comme dans la démonstration de la proposition 4.4 : il suffit d'appliquer l'alternative de Fredholm. Supposons qu'il existe deux solutions $q_{\varepsilon}^{1}$ et $q_{\varepsilon}^{2}$. Posons $v_{\varepsilon}=q_{\varepsilon}^{1}-q_{\varepsilon}^{2}$. Par linéarité de $(23)$, il suffit de montrer que $v_{\varepsilon} \leq 0$, p.p. $x$ de $\Omega$. Sinon, $M_{\varepsilon}=\sup _{x \in \Omega} v_{\varepsilon}(x)>0$. Faisons la différence de $(23)_{q_{\varepsilon}^{1}}$ et $(23)_{q_{\varepsilon}^{2}}$. En multipliant l'équation obtenue, comme dans [15], par la fonction test $\varphi_{\mu}=\left(v_{\varepsilon}-\mu\right)^{+}$, où $\mu$ est un réel tel que $0 \leq \mu<M_{\varepsilon}$, on montre, à l'aide de l'inégalité de Cauchy-Schwartz, que

$$
\left\|\nabla \varphi_{\mu}\right\|_{L^{2}(\Omega)} \leq \frac{2 b}{a^{3} \varepsilon}\left(\int_{\left(v_{\varepsilon}>\mu\right) \cap\left(\nabla v_{\varepsilon} \neq 0\right)} \varphi_{\mu}^{2} d x\right)^{\frac{1}{2}} .
$$

Sachant que l'inclusion de $H_{0}^{1}(\Omega)$ dans $L^{\lambda}(\Omega)$ est continue, si $N \leq 2$, pour tout réel $\lambda \geq 1$, l'estimation (24) et l'inégalité de Hölder entraînent :

$$
\begin{aligned}
\left\|\varphi_{\mu}\right\|_{L^{\lambda}(\Omega)} & \leq C(\Omega, N, \lambda)\left\|\nabla \varphi_{\mu}\right\|_{L^{2}(\Omega)} \leq \frac{2 b}{a^{3} \varepsilon} C(\Omega, N, \lambda)\left(\int_{\Omega} \varkappa_{\left(v_{\varepsilon}>\mu\right) \cap\left(\nabla v_{\varepsilon} \neq 0\right)} \varphi_{\mu}^{2} d x\right)^{\frac{1}{2}} \\
& \leq \frac{2 b}{a^{3} \varepsilon} C(\Omega, N, \lambda)\left\|\varphi_{\mu}\right\|_{L^{\lambda}(\Omega)} \operatorname{mes}\left(\left(v_{\varepsilon}>\mu\right) \cap\left(\nabla v_{\varepsilon} \neq 0\right)\right)^{\frac{1}{2 t}},
\end{aligned}
$$

avec $\lambda>2$ fixé et $\frac{1}{t}+\frac{2}{\lambda}=1$. Par conséquent

$$
\operatorname{mes}\left(\left(v_{\varepsilon}>\mu\right) \cap\left(\nabla v_{\varepsilon} \neq 0\right)\right) \geq c(N, \lambda, \Omega)\left(\frac{a^{3} \varepsilon}{2 b}\right)^{\frac{2 \lambda}{\lambda-2}}
$$


en faisant tendre $\mu$ vers $M_{\varepsilon}$, on obtient

$$
\operatorname{mes}\left(\left(v_{\varepsilon}=M_{\varepsilon}\right) \cap\left(\nabla v_{\varepsilon} \neq 0\right)\right)>0
$$

Ce qui est absurde. En effet (25) implique que la fonction $v_{\varepsilon}$ atteind son maximum au moins en un point $x_{0}$ dans $\Omega$, où elle est dérivable, et $\nabla v_{\varepsilon}\left(x_{0}\right) \neq 0$. Et comme $\Omega$ est un ouvert, on a $\nabla v_{\varepsilon}\left(x_{0}\right)=0$.

Enfin, par différentiation du critère $J_{\varepsilon}$ et du problème $\left(\mathcal{P}_{h}\right)_{\varepsilon}$ par rapport à $h$, la formule de Green implique que :

$$
\begin{aligned}
\left\langle\frac{\partial J_{\varepsilon}\left(h_{\varepsilon}^{*}\right)}{\partial h}, h-h_{\varepsilon}^{*}\right\rangle & =\int_{\Omega}\left(p_{\varepsilon}^{*}-p_{d}\right)\left\langle\frac{\partial p_{\varepsilon}\left(h_{\varepsilon}^{*}\right)}{\partial h}, h-h_{\varepsilon}^{*}\right\rangle+\left(h_{\varepsilon}^{*}-h^{*}\right)\left(h-h_{\varepsilon}^{*}\right) d x \\
& =\int_{\Omega}\left(h-h_{\varepsilon}^{*}\right)\left(H_{\varepsilon}\left(p_{\varepsilon}^{*}\right) \frac{\partial q_{\varepsilon}}{\partial x_{1}}-3 h_{\varepsilon}^{* 2} \nabla p_{\varepsilon}^{*} \nabla q_{\varepsilon}+h_{\varepsilon}^{*}-h^{*}\right) d x
\end{aligned}
$$

et compte tenu de l'optimalité de $h_{\varepsilon}^{*}$ sur le convexe $U_{\text {ad }}$, nous obtenons (22).

\section{Comportement asymptotique du problème $\left(\mathcal{P}_{h_{\varepsilon}}\right)_{\varepsilon}$}

Nous allons montrer maintenant un résultat de convergence forte pour $\left\{p_{\varepsilon}\right\}_{\varepsilon}$ dans $H^{1}(\Omega)$.

Pour cela, on adapte une technique introduite dans [6] qui consiste à faire des estimations sur la solution de $\left(\mathcal{P}_{h}\right)_{\varepsilon}$ dans l'espace de Sobolev $W^{1, s}(\Omega)$, pour un réel $s>2$, indépendamment de $h$.

Dans la suite, nous supposerons l'existence d'un relèvement régulier des conditions aux limites :

$$
\exists \alpha>2, \exists \psi \in W^{1, \alpha}(\Omega) ; \psi \mid \Gamma_{i}=p_{\text {in }} \text { et } \psi \mid \Gamma_{e}=p_{\mathrm{ex}}
$$

Remarque 5.1. Dans la pratique, (voir [7, 10]) le domaine $\Omega$ varie suivant le mécanisme lubrifié considéré. Par exemple:

$\left.\Omega=\Omega^{1}=\right] 0,2 \pi[$ dans le cas du palier unidimensionnel.

$\Omega=\Omega^{2}=\left\{x=\left(x_{1}, x_{2}\right) \in \mathbb{R}^{2} / R_{i}<\sqrt{x_{1}^{2}+x_{2}^{2}}<R_{e}\right\}$ dans le cas du joint d'étanchéité à face radiale, avec $0<R_{i}<R_{e}$.

Il est possible de construire un relèvement régulier $\psi$ des conditions aux limites relativement à chacune de ces configurations du domaine $\Omega$ :

$$
\begin{aligned}
& \psi(x)=\frac{p_{\mathrm{ex}}-p_{\text {in }}}{2 \pi}(x-2 \pi)+p_{\mathrm{ex}} \quad \text { pour } \quad x \in \Omega^{1}, \\
& \psi(x)=\frac{p_{\mathrm{ex}}-p_{\mathrm{in}}}{R_{e}-R_{i}}\left(\sqrt{x_{1}^{2}+x_{2}^{2}}-R_{e}\right)+p_{\mathrm{ex}} \quad \text { pour } \quad\left(x_{1}, x_{2}\right) \in \Omega^{2} .
\end{aligned}
$$

Sous l'hypothèse $\left(H_{1}\right)$, on montre le résultat de régularité suivant :

Proposition 5.2. Si l'hypothèse $\left(H_{1}\right)$ a lieu, alors il existe un réel $s=s(a, b, \alpha, \Omega)$ appartenant à $\left.] 2, \alpha\right]$, tel que pour tout $h$ de $U_{\mathrm{ad}}$, nous avons

$$
\left\|p_{\varepsilon}\right\|_{W^{1, s}(\Omega)} \leq C(a, b, \alpha, s, \Omega)
$$

où $p_{\varepsilon}=p_{\varepsilon}(h)$.

Preuve. Soit $w_{\varepsilon}$ la fonction de $H_{0}^{1}(\Omega)$ définie par $w_{\varepsilon}=p_{\varepsilon}-\psi$, avec $\psi$ vérifiant l'hypothèse $\left(H_{1}\right)$. Comme $0 \leq H_{\varepsilon}\left(p_{\varepsilon}\right) \leq 1, a \leq h \leq b$, presque partout dans $\Omega$, et $p_{\varepsilon}$ est solution de $\left(\mathcal{P}_{h}\right)_{\varepsilon}$, l'inégalité de Hölder 
implique que

$$
\begin{aligned}
\left\|-\operatorname{div}\left(h^{3} \nabla w_{\varepsilon}\right)\right\|_{W^{-1, \alpha}(\Omega)} & \leq\left\|-\operatorname{div}\left(h^{3} \nabla \psi\right)\right\|_{W^{-1, \alpha}(\Omega)}+\left\|\frac{\partial H_{\varepsilon}\left(p_{\varepsilon}\right) h}{\partial x_{1}}\right\|_{W^{-1, \alpha}(\Omega)} \\
& \leq b^{3}\|\nabla \psi\|_{\left(L^{\alpha}(\Omega)\right)^{N}}+b \operatorname{mes}(\Omega)^{1-\frac{1}{\alpha}} \leq C(\alpha, b, \Omega)\left(\|\nabla \psi\|_{\left(L^{\alpha}(\Omega)\right)^{N}}+1\right) ;
\end{aligned}
$$

en utilisant un résultat de régularité (voir par exemple le Th. 4.2 de [8] ou le Th. 7.2 de [18]) on conclut à l'existence d'un réel $\bar{\alpha}=\bar{\alpha}(a, b, \Omega)>2$ tel que pour tout $s$ appartenant à $] 2, \bar{\alpha}]$ :

$$
\forall \varphi \in W_{0}^{1, s}(\Omega),\|\varphi\|_{W_{0}^{1, s}(\Omega)} \leq C_{0}(a, b, s, \Omega)\left\|-\operatorname{div}\left(h^{3} \nabla \varphi\right)\right\|_{W^{-1, s}(\Omega)} .
$$

Donc en particulier, pour $2<s \leq \min (\bar{\alpha}, \alpha)$, on a :

$$
\begin{aligned}
\left\|w_{\varepsilon}\right\|_{W_{0}^{1, s}(\Omega)} & \leq C_{0}(a, b, s, \Omega)\left\|-\operatorname{div}\left(h^{3} \nabla w_{\varepsilon}\right)\right\|_{W^{-1, s}(\Omega)} \leq C_{1}(a, b, s, \alpha, \Omega)\left\|-\operatorname{div}\left(h^{3} \nabla w_{\varepsilon}\right)\right\|_{W^{-1, \alpha}(\Omega)} \\
& \leq C_{2}(a, b, s, \alpha, \Omega)\left(\|\nabla \psi\|_{\left(L^{\alpha}(\Omega)\right)^{N}}+1\right) .
\end{aligned}
$$

Enfin, (26) découle de l'hypothèse $\left(H_{1}\right),(27)$ et de la définition de $w_{\varepsilon}$.

Comme conséquence de (26), on montre :

Proposition 5.3. Soit $\left(h_{\varepsilon}\right)_{\varepsilon}$ une suite d'éléments de $U_{\mathrm{ad}}$ telle que

$$
\begin{gathered}
h_{\varepsilon} \longrightarrow h \quad \text { dans } L^{r}(\Omega), \\
h_{\varepsilon} \longrightarrow h \quad \text { dans } W^{1, r}(\Omega)-\text { faible } ;
\end{gathered}
$$

alors $h$ appartient $\grave{a} U_{\mathrm{ad}}$. Et, à une sous-suite près, nous avons :

$$
\begin{gathered}
h_{\varepsilon} \longrightarrow h \quad \text { dans } L^{\lambda}(\Omega), \forall \lambda \in[1,+\infty[, \\
H_{\varepsilon}\left(p_{\varepsilon}\right) \quad \longrightarrow \quad \text { dans } L^{\infty}(\Omega)-\text { faible }-*, \\
p_{\varepsilon} \quad \longrightarrow \quad \text { dans } W^{1, s}(\Omega)-\text { faible }, \\
p_{\varepsilon} \quad \longrightarrow \quad p \quad \text { dans } C(\bar{\Omega}),
\end{gathered}
$$

où $p_{\varepsilon}=p_{\varepsilon}\left(h_{\varepsilon}\right)$ et $(\theta, p)$ est la solution de $\left(\mathcal{P}_{h}\right)$.

Preuve. Par le même raisonnement que celui de la démonstration de la proposition 3.2, on montre que $h$ appartient à $U_{\mathrm{ad}},(30)$ et (31). D'après la proposition 5.2 , la suite $\left\{p_{\varepsilon}\right\}_{\varepsilon}$ est bornée dans $W^{1, s}(\Omega)$, avec $s>2$, l'inclusion de $W^{1, s}(\Omega)$ dans $C(\bar{\Omega})$ est compacte, donc, à une sous-suite près, on a (32) et (33).

Il reste à montrer que $(\theta, p)$ est solution de $\left(\mathcal{P}_{h}\right)$; on va montrer que $\theta \in H(p)$. Pour le reste, on raisonne comme dans la démonstration de la proposition 3.2. On vérifie, d'après la définition de $H_{\varepsilon}\left(p_{\varepsilon}\right)$, l'estimation suivante

$$
\int_{\Omega}\left(1-H_{\varepsilon}\left(p_{\varepsilon}\right)\right) p_{\varepsilon} d x=\int_{\left(p_{\varepsilon} \leq \varepsilon\right)}\left(1-H_{\varepsilon}\left(p_{\varepsilon}\right)\right) p_{\varepsilon} d x \leq \varepsilon \operatorname{mes}(\Omega)
$$


et sachant que $0 \leq H_{\varepsilon}\left(p_{\varepsilon}\right) \leq 1$ et $p_{\varepsilon} \geq 0$, presque partout dans $\Omega$, on montre, par passage à la limite sur $\varepsilon$, que

$$
\int_{\Omega}(1-\theta) p d x \leq 0,0 \leq \theta \leq 1 \text { et } p \geq 0, \text { p.p. dans } \Omega ;
$$

par conséquent

$$
(1-\theta) p=0,0 \leq \theta \leq 1 \text { et } p \geq 0, \text { p.p. dans } \Omega ;
$$

et donc $\theta \in H(p)$.

Nous allons maintenant énoncer deux lemmes utiles pour la suite :

Lemme 5.4. Soit $\lambda$ appartenant $\grave{a}[1, \infty]$ et $v$ une function de $W^{1, \lambda}(\Omega) ;$ alors

$$
\kappa_{(v=0)} \nabla v=0, \quad \text { p.p. dans } \Omega \text {. }
$$

Preuve. Voir le lemme A.4 de [19].

Lemme 5.5. Avec les notations précédentes, nous avons

$$
\begin{gathered}
H_{\varepsilon}\left(p_{\varepsilon}\right) \longrightarrow 1 \text { dans } L^{\sigma}\left(\Omega_{+}\right), \forall \sigma \in[1,+\infty[, \\
\int_{\Omega}\left|\nabla\left(p_{\varepsilon}-\varepsilon\right)^{-}\right|^{2} d x \longrightarrow 0
\end{gathered}
$$

quand $\varepsilon$ tend vers 0.

Preuve. Montrons d'abord (34). Comme $0 \leq H_{\varepsilon}\left(p_{\varepsilon}\right) \leq 1$ et $0 \leq \theta \leq 1$, presque partout dans $\Omega, \theta=1$, p.p. dans $\Omega_{+}$, nous avons

$$
\left\|1-H_{\varepsilon}\left(p_{\varepsilon}\right)\right\|_{L^{\sigma}\left(\Omega_{+}\right)} \leq \int_{\Omega}\left(\theta-H_{\varepsilon}\left(p_{\varepsilon}\right)\right) \kappa_{\Omega_{+}} d x
$$

compte tenu de (31), le terme de droite dans l'inégalité ci-dessous converge vers 0 , quand $\varepsilon$ tend vers 0 , et donc la conclusion (34) est prouvée. Il reste à montrer (35). En utilisant dans la définition de $\left(\mathcal{P}_{h_{\varepsilon}}\right)_{\varepsilon}$ la fonction test $\left(p_{\varepsilon}-\varepsilon\right)^{-}-(p-\varepsilon)^{-}$, qui appartient à $H_{0}^{1}(\Omega)$, et du fait que $0 \leq H_{\varepsilon}\left(p_{\varepsilon}\right) \leq 1$ et $h_{\varepsilon} \leq b$, presque partout dans $\Omega$, on montre :

$$
\begin{aligned}
R_{\varepsilon}^{0}:= & \int_{\Omega} h_{\varepsilon}^{3}\left|\nabla\left(p_{\varepsilon}-\varepsilon\right)^{-}\right|^{2} d x \\
= & -\int_{\Omega} h_{\varepsilon}^{3} \nabla(p-\varepsilon)^{-} \nabla p_{\varepsilon}+h_{\varepsilon} H_{\varepsilon}\left(p_{\varepsilon}\right) \frac{\partial(p-\varepsilon)^{-}}{\partial x_{1}}-h_{\varepsilon} H_{\varepsilon}\left(p_{\varepsilon}\right) \frac{\partial\left(p_{\varepsilon}-\varepsilon\right)^{-}}{\partial x_{1}} d x \\
\leq & b^{3}\left\|\nabla p_{\varepsilon}\right\|_{\left(L^{2}(\Omega)\right)^{N}}\left\|\kappa_{(p \leq \varepsilon)} \nabla p\right\|_{\left(L^{2}(\Omega)\right)^{N}}+b\left\|\kappa_{(p \leq \varepsilon)} \frac{\partial p}{\partial x_{1}}\right\|_{L^{1}(\Omega)} \\
& +\left|\int_{\Omega} h_{\varepsilon} H_{\varepsilon}\left(p_{\varepsilon}\right) \frac{\partial\left(p_{\varepsilon}-\varepsilon\right)^{-}}{\partial x_{1}} d x\right| ;
\end{aligned}
$$

posons $\varphi_{\varepsilon}(r)=\int_{0}^{r} H_{\varepsilon}(s) d s$. Comme $H_{\varepsilon}\left(p_{\varepsilon}\right)=H_{\varepsilon}\left(\varepsilon-\left(p_{\varepsilon}-\varepsilon\right)^{-}\right)$, la formule de Green implique :

$$
\begin{aligned}
R_{\varepsilon}^{1} & :=\int_{\Omega} h_{\varepsilon} H_{\varepsilon}\left(p_{\varepsilon}\right) \frac{\partial\left(p_{\varepsilon}-\varepsilon\right)^{-}}{\partial x_{1}} d x=\int_{\Omega} h_{\varepsilon} H_{\varepsilon}\left(\varepsilon-\left(p_{\varepsilon}-\varepsilon\right)^{-}\right) \frac{\partial\left(p_{\varepsilon}-\varepsilon\right)^{-}}{\partial x_{1}} d x=-\int_{\Omega} h_{\varepsilon} \frac{\partial \varphi_{\varepsilon}\left(\varepsilon-\left(p_{\varepsilon}-\varepsilon\right)^{-}\right)}{\partial x_{1}} d x \\
& =-\int_{\Gamma} h_{\varepsilon} \varphi_{\varepsilon}\left(\varepsilon-\left(p_{\varepsilon}-\varepsilon\right)^{-}\right) \nu_{1} d \sigma+\int_{\Omega} \frac{\partial h_{\varepsilon}}{\partial x_{1}} \varphi_{\varepsilon}\left(\varepsilon-\left(p_{\varepsilon}-\varepsilon\right)^{-}\right) d x
\end{aligned}
$$


où $\nu_{1}=\cos \left(\nu, x_{1}\right)$ et $\nu$ la dérivée normale. Compte tenu de ce que $0 \leq H_{\varepsilon} \leq 1$, on a pour tout $z \geq 0,0 \leq$ $\varphi_{\varepsilon}(z) \leq z ;$ donc en particulier

$$
0 \leq \varphi_{\varepsilon}\left(\varepsilon-\left(p_{\varepsilon}-\varepsilon\right)^{-}\right) \leq \varepsilon-\left(p_{\varepsilon}-\varepsilon\right)^{-} \leq \varepsilon ;
$$

et comme $\left\|\frac{\partial h_{\varepsilon}}{\partial x_{1}}\right\|_{L^{r}(\Omega)} \leq c$, l'inégalité de Hölder entraîne :

$$
\left|R_{\varepsilon}^{1}\right| \leq \varepsilon\left(\left\|\frac{\partial h_{\varepsilon}}{\partial x_{1}}\right\|_{L^{1}(\Omega)}+b \sigma(\Gamma)\right) \leq \varepsilon\left(\left\|\frac{\partial h_{\varepsilon}}{\partial x_{1}}\right\|_{L^{r}(\Omega)} \operatorname{mes}(\Omega)^{1-\frac{1}{r}}+b \sigma(\Gamma)\right) \leq \varepsilon\left(c \operatorname{mes}(\Omega)^{1-\frac{1}{r}}+b \sigma(\Gamma)\right) ;
$$

la suite $\left(p_{\varepsilon}\right)_{\varepsilon}$ est bornée dans $H^{1}(\Omega)$, donc

$$
\begin{aligned}
\int_{\Omega}\left|\nabla\left(p_{\varepsilon}-\varepsilon\right)^{-}\right|^{2} d x & \leq \frac{R_{\varepsilon}^{0}}{a^{3}} \leq \frac{1}{a^{3}} \times\left(b^{3}\left\|\nabla p_{\varepsilon}\right\|_{L^{2}(\Omega)^{N}}\left\|\kappa_{(p \leq \varepsilon)} \nabla p\right\|_{L^{2}(\Omega)^{N}}+b\left\|\kappa_{(p \leq \varepsilon)} \frac{\partial p}{\partial x_{1}}\right\|_{L^{1}(\Omega)}+\left|R_{\varepsilon}^{1}\right|\right) \\
& \leq C\left(\varepsilon+\left\|\kappa_{(p \leq \varepsilon)} \nabla p\right\|_{L^{2}(\Omega)^{N}}+\left\|\kappa_{(p \leq \varepsilon)} \frac{\partial p}{\partial x_{1}}\right\|_{L^{1}(\Omega)}\right)
\end{aligned}
$$

il suffit maintenant de montrer que le terme de droite de l'inégalité ci-dessous converge vers 0 , quand $\varepsilon$ tend vers 0 . Sachant que $p$ appartient à $H^{1}(\Omega)$ et $p \geq 0$, presque partout dans $\Omega$, nous avons

$$
\left\|\kappa_{(p \leq \varepsilon)} \nabla p\right\|_{L^{2}(\Omega)^{N}}+\left\|\kappa_{(p \leq \varepsilon)} \frac{\partial p}{\partial x_{1}}\right\|_{L^{1}(\Omega)} \rightarrow\left\|\kappa_{(p=0)} \nabla p\right\|_{L^{2}(\Omega)^{N}}+\left\|\kappa_{(p=0)} \frac{\partial p}{\partial x_{1}}\right\|_{L^{1}(\Omega)} .
$$

Enfin, en appliquant le lemme 5.4, nous aboutisserons à la conclusion (35).

Remarque 5.6. Si les pressions d'alimentation $p_{\text {in }}$ et $p_{\mathrm{ex}}$ sont dans $\mathbb{R}_{+}^{*}$, alors la fonction $v_{\varepsilon}=\left(p_{\varepsilon}-\varepsilon\right)^{-}$ appartient à $H_{0}^{1}(\Omega)$, pour $\varepsilon<\min \left(p_{\mathrm{in}}, p_{\mathrm{ex}}\right)$. On fait le même raisonnement avec $v_{\varepsilon}$, on obtient

$$
\int_{\Omega}\left|\nabla\left(p_{\varepsilon}-\varepsilon\right)^{-}\right|^{2} d x \leq C(a, b, c, \Omega, r) \varepsilon .
$$

On peut maintenant montrer le résultat de convergence forte suivant :

Proposition 5.7. Compte tenu de l'hypothèse $\left(H_{1}\right),(28)$ et (29) impliquent qu'à une sous-suite près,

$$
p_{\varepsilon} \longrightarrow p \text { dans } H^{1}(\Omega)
$$

quand $\varepsilon$ tend vers 0 .

Preuve. On vérifie facilement que :

$$
\int_{\Omega} h_{\varepsilon}^{3}\left|\nabla\left(p_{\varepsilon}-p\right)\right|^{2} d x=\int_{\Omega}\left(h^{3}-h_{\varepsilon}^{3}\right) \nabla\left(p_{\varepsilon}-p\right) . \nabla p d x+\int_{\Omega}\left(h_{\varepsilon} H_{\varepsilon}\left(p_{\varepsilon}\right)-\theta h\right) \frac{\partial\left(p_{\varepsilon}-p\right)}{\partial x_{1}} .
$$

Le premier terme de (36) à droite de l'égalité converge vers 0 . En effet, il suffit d'établir, compte tenu de la proposition 5.2, par l'inégalité de Hölder, que

$$
\begin{aligned}
\left|\int_{\Omega}\left(h^{3}-h_{\varepsilon}^{3}\right) \nabla\left(p_{\varepsilon}-p\right) . \nabla p d x\right| & \leq\left\|h^{3}-h_{\varepsilon}^{3}\right\|_{L^{\lambda}(\Omega)}\left\|\nabla\left(p_{\varepsilon}-p\right)\right\|_{L^{s}(\Omega)}\|\nabla p\|_{L^{s}(\Omega)} \\
& \leq 3 b^{2}\left\|h-h_{\varepsilon}\right\|_{L^{\lambda}(\Omega)}\left\|\nabla\left(p_{\varepsilon}-p\right)\right\|_{L^{s}(\Omega)}\|\nabla p\|_{L^{s}(\Omega)} \\
& \leq C(a, b, s, \alpha, \Omega)\left\|h-h_{\varepsilon}\right\|_{L^{\lambda}(\Omega)},
\end{aligned}
$$


avec $\frac{1}{\lambda}+\frac{2}{s}=1$, et d'utiliser (30). Il reste à montrer que le second terme converge aussi vers 0 . En utilisant le lemme 5.4 , on vérifie que

$$
\int_{\Omega}\left(h_{\varepsilon} H_{\varepsilon}\left(p_{\varepsilon}\right)-\theta h\right) \frac{\partial\left(p_{\varepsilon}-p\right)}{\partial x_{1}}=\int_{\Omega_{+}}\left(h_{\varepsilon} H_{\varepsilon}\left(p_{\varepsilon}\right)-h\right) \frac{\partial\left(p_{\varepsilon}-p\right)}{\partial x_{1}} d x+\int_{\Omega_{0}}\left(h_{\varepsilon} H_{\varepsilon}\left(p_{\varepsilon}\right)-\theta h\right) \frac{\partial p_{\varepsilon}}{\partial x_{1}} d x ;
$$

nous allons montrer que les deux termes de (37) à droite convergent vers 0 , quand $\varepsilon$ tend 0 . Pour le premier terme, il suffit d'établir que

$$
\begin{aligned}
W_{\varepsilon} & :=\left|\int_{\Omega_{+}}\left(h_{\varepsilon} H_{\varepsilon}\left(p_{\varepsilon}\right)-h\right) \frac{\partial\left(p_{\varepsilon}-p\right)}{\partial x_{1}} d x\right| \\
& =\left|\int_{\Omega_{+}}\left(h_{\varepsilon}-h\right) H_{\varepsilon}\left(p_{\varepsilon}\right) \frac{\partial\left(p_{\varepsilon}-p\right)}{\partial x_{1}} d x+h\left(H_{\varepsilon}\left(p_{\varepsilon}\right)-1\right) \frac{\partial\left(p_{\varepsilon}-p\right)}{\partial x_{1}} d x\right| \\
& \leq\left\|\frac{\partial\left(p_{\varepsilon}-p\right)}{\partial x_{1}}\right\|_{L^{2}\left(\Omega_{+}\right)}\left(\left\|h_{\varepsilon}-h\right\|_{L^{2}\left(\Omega_{+}\right)}+b\left\|H_{\varepsilon}\left(p_{\varepsilon}\right)-1\right\|_{L^{2}\left(\Omega_{+}\right)}\right) \\
& \leq C\left(\left\|h_{\varepsilon}-h\right\|_{L^{2}(\Omega)}+\left\|H_{\varepsilon}\left(p_{\varepsilon}\right)-1\right\|_{L^{2}\left(\Omega_{+}\right)}\right),
\end{aligned}
$$

et d'utiliser (30) et (34). Quant au second terme, on vérifie d'abord que

$$
\begin{aligned}
Z_{\varepsilon} & :=\left|\int_{\Omega_{0}}\left(h_{\varepsilon} H_{\varepsilon}\left(p_{\varepsilon}\right)-\theta h\right) \frac{\partial p_{\varepsilon}}{\partial x_{1}} d x\right| \\
& =\left|\int_{\Omega_{0} \cap\left(p_{\varepsilon} \geq \varepsilon\right)}\left(h_{\varepsilon}-\theta h\right) \frac{\partial p_{\varepsilon}}{\partial x_{1}} d x+\int_{\Omega_{0} \cap\left(p_{\varepsilon}<\varepsilon\right)}\left(h_{\varepsilon} H_{\varepsilon}\left(p_{\varepsilon}\right)-\theta h\right) \frac{\partial p_{\varepsilon}}{\partial x_{1}} d x\right| \\
& =\left|\int_{\Omega_{0}}\left(h_{\varepsilon}-\theta h\right) \frac{\partial\left(p_{\varepsilon}-\varepsilon\right)^{+}}{\partial x_{1}} d x-\int_{\Omega_{0}}\left(h_{\varepsilon} H_{\varepsilon}\left(p_{\varepsilon}\right)-\theta h\right) \frac{\partial\left(p_{\varepsilon}-\varepsilon\right)^{-}}{\partial x_{1}} d x\right| ;
\end{aligned}
$$

comme

$$
\left|\int_{\Omega_{0}}\left(h_{\varepsilon} H_{\varepsilon}\left(p_{\varepsilon}\right)-\theta h\right) \frac{\partial\left(p_{\varepsilon}-\varepsilon\right)^{-}}{\partial x_{1}} d x\right| \leq 2 b\left\|\frac{\partial\left(p_{\varepsilon}-\varepsilon\right)^{-}}{\partial x_{1}}\right\|_{L^{2}\left(\Omega_{0}\right)} \leq 2 b\left\|\frac{\partial\left(p_{\varepsilon}-\varepsilon\right)^{-}}{\partial x_{1}}\right\|_{L^{2}(\Omega)},
$$

on a, en vertu de (35),

$$
\int_{\Omega_{0}} \frac{\partial\left(p_{\varepsilon}-\varepsilon\right)^{-}}{\partial x_{1}}\left(h_{\varepsilon} H_{\varepsilon}\left(p_{\varepsilon}\right)-\theta h\right) d x \longrightarrow 0 .
$$

En utilisant le fait que $p \geq 0$, p.p. dans $\Omega$, et que la suite $\left\{p_{\varepsilon}\right\}$ converge vers $p$ dans $H^{1}(\Omega)-$ faible et $C(\bar{\Omega})$, on montre que

$$
\frac{\partial\left(p_{\varepsilon}-\varepsilon\right)^{+}}{\partial x_{1}} \longrightarrow \frac{\partial p}{\partial x_{1}} \text { dans } L^{2}(\Omega)-\text { faible }
$$

il en résulte, compte tenu de (30), que

$$
\int_{\Omega_{0}}\left(h_{\varepsilon}-\theta h\right) \frac{\partial\left(p_{\varepsilon}-\varepsilon\right)^{+}}{\partial x_{1}} d x \rightarrow \int_{\Omega_{0}} h(1-\theta) \frac{\partial p}{\partial x_{1}} d x ;
$$


d'après le lemme 5.4, nous avons

$$
\int_{\Omega_{0}} h(1-\theta) \frac{\partial p}{\partial x_{1}} d x=0
$$

Il s'ensuit que le second terme de droite dans (37) converge aussi vers 0. Ce qui achève la démonstration de la proposition.

\section{Conditions nécessaires d’optimalité pour le problème $(\mathcal{M})$ DANS LE CAS UNIDIMENSIONNEL}

Dans cette section, nous allons nous restreindre au cas unidimensionnel pour lequel nous pouvons établir des estimations sur l'état adjoint. La difficulté est essentiellement liée au terme $-h_{\varepsilon}^{*} \dot{H}_{\varepsilon}\left(p_{\varepsilon}^{*}\right) \frac{\partial q_{\varepsilon}}{\partial x_{1}}$, figurant dans l'équation (23). Pour simplifier, on considère le domaine $\Omega=] 0,1[$. On commence par montrer que la suite $\left(q_{\varepsilon}\right)_{\varepsilon}$ est bornée dans $L^{\infty}(] 0,1[)$. Pour cela, on supposera que

$$
p_{d} \in L^{\infty}(] 0,1[)
$$

Proposition 6.1. Sous l'hypothèse $\left(H_{2}\right)$, nous avons

$$
\left\|q_{\varepsilon}\right\|_{L^{\infty}(] 0,1[)} \leq \frac{1}{2 a^{3}}\left\|p_{\varepsilon}^{*}-p_{d}\right\|_{\left.\left.L^{\infty}(] 0,1\right]\right)} .
$$

Preuve. On pose

$$
Q=q_{\varepsilon}+C w, \text { avec } w(x)=\int_{0}^{x} \frac{y}{h_{\varepsilon}^{* 3}(y)} d y
$$

remarquons que

$$
h_{\varepsilon}^{* 3}(x) \frac{d w}{d x}=x \text { et } \frac{d}{d x}\left(h_{\varepsilon}^{* 3} \frac{d w}{d x}\right)=1
$$

ce qui implique

$$
-\frac{d}{d x}\left(h_{\varepsilon}^{* 3} \frac{d Q}{d x}\right)-h_{\varepsilon}^{*} \dot{H}\left(p_{\varepsilon}^{*}\right) \frac{d Q}{d x}=F_{\varepsilon},
$$

avec

$$
F_{\varepsilon}=-\frac{d}{d x}\left(h_{\varepsilon}^{* 3} \frac{d q_{\varepsilon}}{d x}\right)-h_{\varepsilon}^{*} \dot{H}\left(p_{\varepsilon}^{*}\right) \frac{d q_{\varepsilon}}{d x}-C\left(1+h_{\varepsilon}^{*} \dot{H}\left(p_{\varepsilon}^{*}\right) \frac{d w}{d x}\right)=p_{\varepsilon}^{*}-p_{d}-C\left(1+\frac{x \dot{H}\left(p_{\varepsilon}^{*}\right)}{h_{\varepsilon}^{* 2}}\right)
$$

Notons d'abord que $\left(F_{\varepsilon}, Q\right)$ appartient à $L^{2}(] 0,1[) \times H^{1}(] 0,1[)$. Choisissons $C=\left\|p_{\varepsilon}^{*}-p_{d}\right\|_{L^{\infty}(] 0,1[)}$; alors $F_{\varepsilon} \leq 0$. Donc en appliquant le principe du maximum, on obtient $Q \leq \sup Q$. Ce qui entraîne $\{0,1\}$

$$
q_{\varepsilon}(x)=Q(x)-C w(x) \leq \sup _{\{0,1\}} Q-C w(x) \leq\left\|p_{\varepsilon}^{*}-p_{d}\right\|_{L^{\infty}(] 0,1[)} \int_{x}^{1} \frac{y}{h_{\varepsilon}^{* 3}(y)} d y
$$


pour presque tout $x$ de $] 0,1\left[\right.$; d'autre part, le choix $C=-\left\|p_{\varepsilon}^{*}-p_{d}\right\|_{L^{\infty}(] 0,1[)}$, implique $F_{\varepsilon} \geq 0$. En répétant le même raisonnement, on montre que

$$
q_{\varepsilon}(x) \geq-\left\|p_{\varepsilon}^{*}-p_{d}\right\|_{L^{\infty}(] 0,1[)} \int_{x}^{1} \frac{y}{h_{\varepsilon}^{* 3}(y)} d y ;
$$

il en résulte que

$$
\left|q_{\varepsilon}(x)\right| \leq\left\|p_{\varepsilon}^{*}-p_{d}\right\|_{\left.\left.L^{\infty}(] 0,1\right]\right)} \int_{x}^{1} \frac{y}{h_{\varepsilon}^{* 3}(y)} d y \leq \frac{(x-1)^{2}}{2 a^{3}}\left\|p_{\varepsilon}^{*}-p_{d}\right\|_{\left.\left.L^{\infty}(] 0,1\right]\right)} \leq \frac{1}{2 a^{3}}\left\|p_{\varepsilon}^{*}-p_{d}\right\|_{\left.\left.L^{\infty}(] 0,1\right]\right)},
$$

presque partout dans ]0, 1 [; ce qui prouve l'estimation (38).

Dans la suite, on pose $\mu_{\varepsilon}=h_{\varepsilon}^{*} \dot{H}_{\varepsilon}\left(p_{\varepsilon}^{*}\right) \frac{d q_{\varepsilon}}{d x}$. Montrons le lemme suivant :

Lemme 6.2. Avec les notations précédentes, nous avons

$$
\int_{0}^{1}\left|\mu_{\varepsilon}\right| \zeta d x=\int_{0}^{1} h_{\varepsilon}^{* 3}\left|\frac{d q_{\varepsilon}}{d x}\right| \frac{d \zeta}{d x}-\operatorname{sign}_{0}\left(\frac{d q_{\varepsilon}}{d x}\right)\left(p_{\varepsilon}^{*}-p_{d}\right) \zeta d x, \forall \zeta \in H_{0}^{1}(] 0,1[)
$$

où la fonction $\operatorname{sign}_{0}$ est définie par

$$
\operatorname{sign}_{0}(z)=\left\{\begin{array}{ccc}
1 & \text { si } & z>0 \\
0 & \text { si } & z=0 \\
-1 & \text { si } & z<0
\end{array}\right.
$$

Preuve. Soit $\beta_{\sigma}$ la régularisation de la fonction $\operatorname{sign}_{0}$ définie par

$$
\beta_{\sigma}(z)=\left\{\begin{array}{lll}
1 & \text { si } & z>\sigma \\
\frac{z}{\sigma} & \text { si } & -\sigma \leq z \leq \sigma \\
-1 & \text { si } & z<-\sigma
\end{array}\right.
$$

avec $\sigma>0$. L'équation d'état adjointe s'écrit, compte tenu de la définition de $\mu_{\varepsilon}$,

$$
\left\{\begin{array}{l}
q_{\varepsilon} \in H_{0}^{1}(] 0,1[) \\
-\frac{d}{d x}\left(h_{\varepsilon}^{* 3} \frac{d q_{\varepsilon}}{d x}\right)=\mu_{\varepsilon}+p_{\varepsilon}^{*}-p_{d}
\end{array}\right.
$$

comme le second membre $\mu_{\varepsilon}+p_{\varepsilon}^{*}-p_{d}$ et le coefficient $h_{\varepsilon}^{*}$ appartiennent respectivement à $L^{2}(] 0,1[)$ et $W^{1, r}(] 0,1[)$, avec $r>1$, on a, dans le cas $N=1$, d'après un résultat de régularité pour les problèmes elliptiques linéaires (voir par exemple le Th. 10.1 de [16])

$$
q_{\varepsilon} \in H^{2}(] 0,1[) \cap H_{0}^{1}(] 0,1[)
$$

d'où

$$
v_{\varepsilon}=\frac{d q_{\varepsilon}}{d x} \in H^{1}(] 0,1[) \text { et } \beta_{\sigma}\left(v_{\varepsilon}\right) \in H^{1}(] 0,1[) .
$$

L'inclusion de $H^{1}(] 0,1[)$ dans $L^{\infty}(] 0,1[)$ implique que

$$
\zeta \beta_{\sigma}\left(v_{\varepsilon}\right) \in H_{0}^{1}(] 0,1[),
$$


pour toute fonction $\zeta$ dans $H_{0}^{1}(] 0,1[)$. On peut alors prendre $\zeta \beta_{\sigma}\left(v_{\varepsilon}\right)$ comme fonction test dans (39) :

$$
\int_{0}^{1} \mu_{\varepsilon} \beta_{\sigma}\left(v_{\varepsilon}\right) \zeta d x=\int_{0}^{1} \beta_{\sigma}\left(v_{\varepsilon}\right)\left(h_{\varepsilon}^{* 3} v_{\varepsilon} \frac{d \zeta}{d x}-\left(p_{\varepsilon}^{*}-p_{d}\right) \zeta\right) d x+\int_{0}^{1} h_{\varepsilon}^{* 3} v_{\varepsilon} \dot{\beta}_{\sigma}\left(v_{\varepsilon}\right) \zeta \frac{d v_{\varepsilon}}{d x} d x .
$$

On va montrer que le second terme de (40) à droite converge vers 0 , quand $\sigma$ tend vers 0 . Le passage à la limite dans les autres termes est classique. Comme $\left|\dot{\beta}_{\sigma}\left(v_{\varepsilon}\right) v_{\varepsilon}\right| \leq 1$, nous avons

$$
\left|\int_{0}^{1} h_{\varepsilon}^{* 3} v_{\varepsilon} \dot{\beta}_{\sigma}\left(v_{\varepsilon}\right) \zeta \frac{d v_{\varepsilon}}{d x}\right| \leq b^{3} \int_{\left(\left|v_{\varepsilon}\right| \leq \sigma\right)}\left|\dot{\beta}_{\sigma}\left(v_{\varepsilon}\right) v_{\varepsilon}\right|\left|\zeta \frac{d v_{\varepsilon}}{d x}\right| d x \leq b^{3} \int_{\left(\left|v_{\varepsilon}\right| \leq \sigma\right)}\left|\zeta \frac{d v_{\varepsilon}}{d x}\right| d x
$$

l'intégrale de droite dans l'inégalité ci-dessous converge vers $\int_{\left(v_{\varepsilon}=0\right)}\left|\zeta \frac{d v_{\varepsilon}}{d x}\right| d x$. En vertu du lemme 5.4, on a $\int_{\left(v_{\varepsilon}=0\right)}\left|\zeta \frac{d v_{\varepsilon}}{d x}\right| d x=0$, et donc

$$
\int_{0}^{1} h_{\varepsilon}^{* 3} v_{\varepsilon} \dot{\beta}_{\sigma}\left(v_{\varepsilon}\right) \zeta \frac{d v_{\varepsilon}}{d x} \longrightarrow 0
$$

Enfin, par passage à la limite sur $\sigma$ dans (40), et compte tenu de (41), nous aboutissons à la conclusion du lemme.

On va établir d'autres estimations permettant de passer à la limite dans les conditions nécessaires d'optimalité pour le problème régularisé. Pour cela, considérons l'espace affine

$$
C_{A}=\left\{\varphi \in C([0,1]) / \varphi(0)=p_{\text {in }} \text { et } \varphi(1)=p_{\text {ex }}\right\} .
$$

Et soit $C^{+}$le cône positif de $C_{A}$ défini par

$$
C^{+}=\left\{\varphi \in C_{A} / \varphi \geq 0\right\} .
$$

Dans la suite, on supposera que

$$
\left(p_{\text {in }}, p_{\text {ex }}\right) \in \mathbb{R}_{+}^{*} \times \mathbb{R}_{+}^{*} .
$$

Sous cette hypothèse, on montre que $C^{+}$est d'intérieur non vide :

Lemme 6.3. Sous l'hypothèse $\left(H_{3}\right)$, pour toute fonction $\varphi$ dans $C^{+}$vérifiant $\inf _{[0,1]} \varphi>0$, il existe un réel $\rho>0$ tel que

$$
\varphi+\rho \mathbb{B}(0,1) \subset C^{+}
$$

où $\mathbb{B}(0,1)$ est la boule unité de $C_{0}([0,1])$.

Preuve. Il suffit de prendre $\rho$ vérifiant $0<\rho<\inf _{[0,1]} \varphi$.

Nous pouvons maintenant montrer le résultat suivant :

Proposition 6.4. Il existe une constante $C$ strictement positive telle que pour tout $\varepsilon>0$, nous avons:

$$
\left\|\mu_{\varepsilon}\right\|_{L^{1}(] 0,1[)}+\left\|\mu_{\varepsilon}\right\|_{H^{-1}(] 0,1[)} \leq C .
$$


Preuve. Posons, comme dans [11,12],

$$
\alpha_{\varepsilon}=\frac{1}{1+\left\|\mu_{\varepsilon}\right\|_{L^{1}(] 0,1[)}+\left\|\mu_{\varepsilon}\right\|_{H^{-1}(] 0,1[)}}, \bar{q}_{\varepsilon}=\alpha_{\varepsilon} q_{\varepsilon} \text { et } \bar{\mu}_{\varepsilon}=\alpha_{\varepsilon} \mu_{\varepsilon} ;
$$

il vient

$$
\begin{gathered}
\alpha_{\varepsilon}+\left\|\bar{\mu}_{\varepsilon}\right\|_{L^{1}(] 0,1[)}+\left\|\bar{\mu}_{\varepsilon}\right\|_{H^{-1}(] 0,1[)}=1, \\
-\frac{d}{d x}\left(h_{\varepsilon}^{* 3} \frac{d \bar{q}_{\varepsilon}}{d x}\right)=\bar{\mu}_{\varepsilon}+\alpha_{\varepsilon}\left(p_{\varepsilon}^{*}-p_{d}\right) .
\end{gathered}
$$

Raisonnons par l'absurde en supposant que $\left\{\mu_{\varepsilon}\right\}$ n'est pas bornée dans $L^{1}((] 0,1[)) \cap H^{-1}(] 0,1[)$. Alors, à une sous-suite près, $\alpha_{\varepsilon}$ converge vers 0 , quand $\varepsilon$ tend vers 0 . On montre aisément, comme les suites $\left\{q_{\varepsilon}\right\},\left\{\bar{\mu}_{\varepsilon}+\alpha_{\varepsilon}\left(p_{\varepsilon}^{*}-p_{d}\right)\right\}$ et $\left\{p_{\varepsilon}^{*}-p_{d}\right\}$ sont bornées respectivement dans $L^{\infty}(] 0,1[), L^{1}(] 0,1[)$ et $H^{-1}(] 0,1[)$, les estimations suivantes

$$
\begin{gathered}
\left\|\bar{q}_{\varepsilon}\right\|_{H_{0}^{1}(] 0,1[)} \leq C \alpha_{\varepsilon}, \\
\left\|\bar{\mu}_{\varepsilon}\right\|_{H^{-1}(] 0,1[)} \leq C\left(\alpha_{\varepsilon}+\left\|\bar{q}_{\varepsilon}\right\|_{H_{0}^{1}(] 0,1[)}\right) ;
\end{gathered}
$$

comme $\left\{\alpha_{\varepsilon}\right\}$ converge vers 0 , il en résulte que les suites $\left\{\bar{q}_{\varepsilon}\right\}$ et $\left\{\bar{\mu}_{\varepsilon}\right\}$ convergent vers 0 , respectivement dans les espaces $H_{0}^{1}(] 0,1[)$ et $H^{-1}(] 0,1[)$. Et donc, avec (42), nous avons

$$
\left\|\bar{\mu}_{\varepsilon}\right\|_{L^{1}(] 0,1[)} \longrightarrow 1
$$

Soit $\varphi$ appartenant à $H^{1}(\Omega) \cap C^{+}$et vérifiant l'hypothèse du lemme 6.3 . Alors il existe $\rho>0$, tel que pour tout $u$ dans $\mathbb{B}(0,1), \varphi-\rho u$ appartient à $C^{+}$; et comme $\left\|\bar{\mu}_{\varepsilon}\right\|_{L^{1}(] 0,1[)} \leq 1$, nous avons

$$
\begin{aligned}
\int_{0}^{1}\left|\bar{\mu}_{\varepsilon}\right|\left(p_{\varepsilon}^{*}-(\varphi-\rho u)\right) d x & =\int_{\left(p_{\varepsilon} \leq \varepsilon\right)}\left|\bar{\mu}_{\varepsilon}\right|\left(p_{\varepsilon}^{*}-(\varphi-\rho u)\right) d x \leq \int_{\left(p_{\varepsilon} \leq \varepsilon\right)}\left|\bar{\mu}_{\varepsilon}\right|(\varepsilon-(\varphi-\rho u)) d x \\
& \leq \varepsilon \int_{\left(p_{\varepsilon} \leq \varepsilon\right)}\left|\bar{\mu}_{\varepsilon}\right| \leq \varepsilon ;
\end{aligned}
$$

ce qui entraîne

$$
\rho \int_{0}^{1}\left|\bar{\mu}_{\varepsilon}\right| u d x \leq \varepsilon+\int_{0}^{1}\left|\bar{\mu}_{\varepsilon}\right|\left(\varphi-p_{\varepsilon}^{*}\right) d x
$$

par conséquent

$$
\begin{aligned}
\rho\left\|\bar{\mu}_{\varepsilon}\right\|_{\left.\left.L^{1}(] 0,1\right]\right)} & =\rho \sup _{u \in \mathbb{B}(0,1)} \int_{0}^{1}\left|\bar{\mu}_{\varepsilon}\right| u d x \leq \varepsilon+\int_{0}^{1}\left|\bar{\mu}_{\varepsilon}\right|\left(\varphi-p_{\varepsilon}^{*}\right) d x \\
& \leq \varepsilon+\int_{0}^{1}\left|\bar{\mu}_{\varepsilon}\right|\left(\varphi-p^{*}\right) d x+\int_{0}^{1}\left|\bar{\mu}_{\varepsilon}\right|\left(p^{*}-p_{\varepsilon}^{*}\right) d x \\
& \leq \varepsilon+\int_{0}^{1}\left|\bar{\mu}_{\varepsilon}\right|\left(\varphi-p^{*}\right) d x+\left\|\bar{\mu}_{\varepsilon}\right\|_{\left.\left.L^{1}(] 0,1\right]\right)}\left\|p^{*}-p_{\varepsilon}^{*}\right\|_{L^{\infty}(] 0,1[)} \\
& \leq \varepsilon+\int_{0}^{1}\left|\bar{\mu}_{\varepsilon}\right|\left(\varphi-p^{*}\right) d x+\left\|p^{*}-p_{\varepsilon}^{*}\right\|_{L^{\infty}(] 0,1[)}
\end{aligned}
$$


$p^{*}$ étant donnée par la proposition 5.3. En prenant $\zeta=\alpha_{\varepsilon}\left(\varphi-p^{*}\right)$ dans le lemme 6.2 , on vérifie, compte tenu de (43), que

$$
\int_{0}^{1}\left|\bar{\mu}_{\varepsilon}\right|\left(\varphi-p^{*}\right) d x \leq C(a, b, \varphi, \Omega)\left(\left\|\bar{q}_{\varepsilon}\right\|_{H_{0}^{1}(] 0,1[)}+\alpha_{\varepsilon}\right) \leq \hat{C}(a, b, \varphi, \Omega) \alpha_{\varepsilon}
$$

et donc l'estimation (45) devient

$$
\rho\left\|\bar{\mu}_{\varepsilon}\right\|_{\left.\left.L^{1}(] 0,1\right]\right)} \leq \varepsilon+\hat{C}_{\varphi} \alpha_{\varepsilon}+\left\|p^{*}-p_{\varepsilon}^{*}\right\|_{\left.\left.L^{\infty}(] 0,1\right]\right)} .
$$

Comme les suites $\left\{p_{\varepsilon}^{*}\right\}$ et $\left\{\alpha_{\varepsilon}\right\}$ convergent respectivement vers $p^{*}$ dans $C([0,1])$ et 0 dans $\mathbb{R},(44)$ implique, par passage à la limite sur $\varepsilon$ dans l'inégalité ci-dessous, que $\rho \leq 0$. Ce qui est absurde car $\rho>0$.

Proposition 6.5. Si $h_{\varepsilon}^{*}$ est un contrôle optimal pour le problème $\left(\mathcal{M}_{\varepsilon}\right)$, alors la suite des états adjoints associés $\left(q_{\varepsilon}\right)_{\varepsilon}$ vérifie les estimations suivantes :

$$
\begin{gathered}
\left\|q_{\varepsilon}\right\|_{L^{\infty}(] 0,1[)}+\left\|\mu_{\varepsilon}\right\|_{L^{1}(] 0,1[)}+\left\|\mu_{\varepsilon}\right\|_{\left.\left.H^{-1}(] 0,1\right]\right)} \leq C, \\
\left\|q_{\varepsilon}\right\|_{\left.\left.H_{0}^{1}(] 0,1\right]\right)} \leq C,
\end{gathered}
$$

où $C$ une constante qui ne dépend pas de $\varepsilon$.

Preuve. L'estimation (46) est une conséquence des propositions 6.1 et 6.4. Quant à (47), elle découle, en écrivant l'équation d'état adjointe sous la forme (39), des estimations classiques pour les problèmes elliptiques linéaires.

Dans le lemme qui va suivre, on reprend les résultats obtenus dans la proposition 5.3. Le terme de pénalisation adaptée $\frac{1}{2} \int_{0}^{1}\left(h-h^{*}\right)^{2} d x$ assure, comme nous l'avons déjà mentionné, la convergence forte dans $L^{2}(] 0,1[)$ des solutions $\left\{h_{\varepsilon}^{*}\right\}$, pour le problème $\left(\mathcal{M}_{\varepsilon}\right)$, vers le contrôle optimale $h^{*}$. Ainsi, on peut vérifier :

Lemme 6.6. A une sous-suite près, nous avons

$$
\begin{array}{lll}
H_{\varepsilon}\left(p_{\varepsilon}^{*}\right) \longrightarrow \theta^{*} & \text { dans } & L^{\infty}(] 0,1[)-\text { faible-* } \\
h_{\varepsilon}^{*} \longrightarrow h^{*} & \text { dans } & L^{\lambda}(] 0,1[), \forall \lambda \in[1, \infty[, \\
p_{\varepsilon}^{*} \longrightarrow p^{*} & \text { dans } & H^{1}(] 0,1[), \\
p_{\varepsilon}^{*} \longrightarrow p^{*} & \text { dans } & W^{1, s}(] 0,1[)-\text { faible et } C([0,1]),
\end{array}
$$

où $\left(\theta^{*}, p^{*}\right)$ est solution de $\left(\mathcal{P}_{h^{*}}\right)$.

On va finalement donner le principal résultat de ce travail qui concerne le cas unidimensionnel. Pour cela, posons

$$
] 0,1\left[+=\{x \in] 0,1\left[/ p^{*}(x)>0, \text { p.p. dans }\right] 0,1[\} .\right.
$$


Sous les hypothèses $\left(H_{1}, H_{2}\right)$ et $\left(H_{3}\right)$, nous avons :

Théorème 6.7. Soit $h^{*}$ un contrôle optimal pour le problème $(\mathcal{M})$. Alors il existe $\left(p^{*}, \theta^{*}, q, \mu\right)$ dans $H^{1}(] 0,1[) \times$ $L^{\infty}(] 0,1[) \times H_{0}^{1}(] 0,1[) \times M(] 0,1[)$ tel que :

$$
\begin{gathered}
\left\{\begin{array}{l}
-\frac{d}{d x}\left(h^{* 3} \frac{d p^{*}}{d x}\right)+\frac{d\left(\theta^{*} h^{*}\right)}{d x}=0 \\
\theta^{*} \in H\left(p^{*}\right) \\
p^{*}(0)=p_{\text {in }} \text { et } p^{*}(1)=p_{\text {ex }},
\end{array}\right. \\
-\frac{d}{d x}\left(h^{* 3} \frac{d q}{d x}\right)=\mu+p^{*}-p_{d}, \\
\left\{\begin{array}{l}
\forall h \in U_{\mathrm{ad}} \\
\int_{0}^{1}\left(h-h^{*}\right)\left(\theta^{*} \frac{d q}{d x}-3 h^{* 2} \frac{d p^{*}}{d x} \frac{d q}{d x}\right) d x \geq 0,
\end{array}\right. \\
\left\langle|\mu|, p^{*}-\varphi\right\rangle_{M\left(10,1[), C_{0}([0,1]) \leq 0, \quad \forall \varphi \in C^{+},\right.}^{\mu} \leq \quad \text { dans } \quad H^{-1}(] 0,1[+),
\end{gathered}
$$

où $|\mu|$ est une limite $M(] 0,1[)-$ faible-* de $\left(\left|\mu_{\varepsilon}\right|\right)_{\varepsilon}$.

Preuve. Les estimations $(46,47)$ impliquent l'existence de deux multiplicateurs $q$ et $\mu$ appartenant respectivement à $H_{0}^{1}(] 0,1[)$ et $M(] 0,1[)$, tels que $\left\{q_{\varepsilon}\right\}$ converge vers $q$ dans $H_{0}^{1}(] 0,1[)$-faible et $\mu_{\varepsilon}$ converge vers $\mu$ dans $M(] 0,1[)$-faible-* et $H^{-1}(] 0,1[)$-faible-*, où $M(] 0,1[)=\left(C_{0}([0,1])\right)^{*}$ est l'espace des mesures bornées sur ]0,1[. Utilisant le lemme 6.6, on peut passer à la limite sur $\varepsilon$ dans (21-23) et aboutir aux conclusions (48-50). Il reste à prouver (51) et (52). Pour une fonction $\varphi$ appartenant à $C^{+}$, l'estimation (46) entraîne que

$$
\int_{0}^{1}\left|\mu_{\varepsilon}\right|\left(p_{\varepsilon}^{*}-\varphi\right) d x=\int_{\left(p_{\varepsilon}^{*} \leq \varepsilon\right)}\left|\mu_{\varepsilon}\right|\left(p_{\varepsilon}^{*}-\varphi\right) d x \leq \varepsilon\left\|\mu_{\varepsilon}\right\|_{L^{1}(] 0,1[)} \leq C \varepsilon ;
$$

et comme $\left\{p_{\varepsilon}^{*}\right\}$ converge vers $p^{*}$ dans $C([0,1])$, on obtient, en faisant tendre $\varepsilon$ vers 0 dans l'estimation (53),

$$
\left\langle|\mu|, p^{*}-\varphi\right\rangle_{M(\Omega), C_{0}([0,1])} \leq 0 .
$$

Soit maintenant $\varphi$ appartenant à $D(] 0,1\left[_{+}\right)$et $\left.K \quad \subset\right] 0,1\left[+\right.$ son support. La suite $\left\{p_{\varepsilon}^{*}\right\}$ converge vers $p^{*}$ dans $C([0,1])$, donc il existe $\varepsilon_{0}>0$ tel que

$$
\forall \varepsilon \leq \min \left(\varepsilon_{0}, \frac{3 d}{2}\right), \forall x \in K, p_{\varepsilon}^{*}(x) \geq p^{*}(x)+\frac{d}{2} \geq \frac{3 d}{2} \geq \varepsilon,
$$

où $d=\min _{K} p^{*}>0$. Par conséquent $\dot{H}_{\varepsilon}\left(p_{\varepsilon}^{*}\right)=0$ sur $K$ et par suite

$$
\left\langle\mu_{\varepsilon}, \varphi\right\rangle=\int_{K} \mu_{\varepsilon} \varphi d x=0
$$


$D(] 0,1[+)$ étant dense dans $H_{0}^{1}(] 0,1[+)$, la convergence de $\left\{\mu_{\varepsilon}\right\}$ vers $\mu$ dans $H^{-1}(] 0,1[)$-faible-*, implique, en faisant tendre $\varepsilon$ vers 0 dans (54), que

$$
\langle\mu, \varphi\rangle=0, \forall \varphi \in H_{0}^{1}(] 0,1[+) .
$$

Ce qui achève la démonstration du théorème.

\section{Conclusions}

Le raisonnement utilisé ici pour dériver les conditions d'optimalité pour le problème $(\mathcal{M})$ n'est pas valable dans le cas bidimensionnel. En effet, si $\varepsilon$ tend vers 0 , il n'est pas évident, que $\alpha+\|\bar{\mu}\|_{M(\Omega)}+\|\bar{\mu}\|_{H^{-1}(\Omega)}>0$, où $\alpha$ est un point d'accumulation de $\left(\alpha_{\varepsilon}\right)_{\varepsilon}$ et $\bar{\mu}$ est une limite faible de $\bar{\mu}_{\varepsilon}$. Or ceci est indispensable pour établir au moins des conditions nécessaires d'optimalité de type Fritz-John [11,13]. On montre que l'état adjoint est borné dans $L^{\infty}(\Omega)$, si on considère des épaisseurs ne dépendant que de la variable spatiale $x_{1}$. Cependant, cela n'est pas suffisant pour pouvoir passer à la limite. Ainsi, le problème dans le cas bidimensionnel reste ouvert.

\section{RÉFÉRENCES}

[1] C. Alvarez, Problemas de frontiera libre en teoría de lubrificación. Ph.D. Thesis, Complutense University of Madrid (1986).

[2] V. Barbu, Necessary conditions for nonconvex distributed control problems governed by elliptic variational inequalities. $J$. Math. Anal. Appl. 80 (1981) 566-598.

[3] V. Barbu, Necessary conditions for distributed control problems governed by parabolic variational inequalities. SIAM. J. Control Optim. 19 (1981) 64-86.

[4] G. Bayada et M. Chambat, Sur quelques modélisation de la zone de cavitation en lubrification hydrodynamique. J. Méc. Théor. Appl. 5 (1986) 703-729.

[5] G. Bayada et M. Chambat, Existence and uniqueness for a lubrification problem with non regular conditions on the free boundary. Boll. Un Math. Ital. 6 (1984) 543-547.

[6] G. Bayada et M. El Alaoui Talibi, Control by coefficients in a variational inequality: The inverse elastohydrodynamic lubrication problem. Nonlinear Analysis: Real World Applications 1 (2000) 315-328.

[7] G. Bayada et M. El Alaoui Talibi, Une méthode du type caractérisitique pour la résolution d'un problème de lubrification hydrodynamique en régime transitoire. ESAIM: M2AN 25 (1991) 395-423.

[8] A. Bensoussan, J.L. Lions et G. Papanicolau, Asymptotic analysis for periodic structures. North-Holland, Amsterdam (1978).

[9] H. Brezis, Analyse fonctionnelle Théorie et Application. Masson, Paris (1983).

[10] A. Cameron, Basic Lubrication Theory. John Whiley \& Sons (1981).

[11] E. Casas et F. Bonnans, An extension of pontryagin's principle for state-constrainted optimal control of semilinear elliptic equations and variational inequalities. SIAM J. Control Optim. 33 (1995) 274-298.

[12] E. Casas et F. Bonnans, Optimal control of semilinear multistate systems with state constraints. SIAM J. Control Optim. 27 (1989) 446-455.

[13] E. Casas, O. Kavian et J.P. Puel, Optimal control of an ill-posed elliptic semilinear equation whith an exponential non linearity. ESAIM: COCV 3 (1998) 361-380.

[14] G. Elrod H. et M.L. Adams, A computer program for cavitation, in st LEEDS LYON symposium on cavitation and related phenomena in lubrication, I.M.E. (1974).

[15] D. Gilbarg et N.S. Trudinger, Elliptic Partial Differential Equations of second Order. Springer-Verlag (1983).

[16] O.A. Ladyzhenskaya et N.N. Ural'tseva, Linear and quasilinear elliptic equations. Academic Press (1968).

[17] M.H. Meurisse, Solution of the inverse problem in hydrodynamic lubrication, in Proc. of the X Lyon Leeds International Symposium (1983) 104-107.

[18] J.F. Rodrigues, Obstacle problems in mathematical physics. North-Holland, Amsterdam (1978).

[19] G. Stampachia et D. Kinderleher, An introduction to variational inequalities and applications. Academic Press (1980). 